\title{
PITCHFORK AND HOPF BIFURCATIONS OF TRAVELING PULSES GENERATED BY COEXISTING FRONT AND BACK WAVES*
}

\author{
SHUNSAKU NII
}

\begin{abstract}
Pitchfork and Hopf bifurcations of traveling pulse solutions bifurcating from coexisting traveling front and back waves of reaction-diffusion systems are studied. It is assumed that the parameter set on which the traveling front exists and the set on which the back wave exists intersect non-transversally. As a result, more complicated bifurcations than the transversal case are proven to occur, including pitchfork and Hopf bifurcations of traveling pulses.
\end{abstract}

1. Introduction. Recently, bifurcation phenomena of traveling waves and their stability in parabolic systems has been attracting attention. Such systems include reaction-diffusion systems, where the waves are thought to represent qualitative change of propagation of transition layers of chemical substances, and nerve axon equations, where they are thought to represent conduction of electric pulses. These waves are expected to be stable if they are observed experimentally. This problem has already commanded a large body of literature.

One way to study this subject is to regard the bifurcations and the linear stability of traveling waves as bifurcations of homoclinic or heteroclinic solutions of ordinary differential equations.

A natural strategy in this line of thought is as follows:

(1) Assume the weakest type of degeneracies on the homoclinic or heteroclinic orbits corresponding to the wave and apply the homoclinic or heteroclinic bifurcation theory. Then analyze the stability of waves corresponding to the homoclinic or heteroclinic solutions.

(2) Proceed to more degenerate cases.

Results in stage (1) go back to early works by Evans, Fenichel and Feroe [5], Yanagida [21], Yanagida and Maginu [22] Kokubu, Nishiura and Oka [13] and Deng [4]. Later results include Alexander and Jones [2][3], Gardner [6], Kan-on [10][11], Nii [14] [15][16] and Sandstede [18][19]. [13] and [14] treat bifurcation of pulses from coexisting front and back waves under certain transversality conditions. Works belonging to stage (2) have already done by Sandstede, Alexander and Jones [20], Yew [23] and Nii and Sandstede [17].

The subject of this paper is bifurcation from coexisting front and back waves which do not satisfy transversality conditions. This type of degeneracy appears in [13] and belongs to stage (2) above.

In [13], the following type of one-dimensional reaction diffusion system is treated:

$$
\left\{\begin{aligned}
\varepsilon \tau u_{t} & =\varepsilon^{2} u_{x x}+f(u, v ; \theta, \gamma) \\
v_{t} & =v_{x x}+g(u, v ; \theta, \gamma)
\end{aligned}\right.
$$

where $x \in \mathbb{R}$ and $t>0 . \varepsilon$ and $\tau$ are real positive parameters, with $\varepsilon$ a small parameter. The nullcline of $f$ intersects with that of $g$ at $P=\left(u_{P}, v_{P}\right), R=\left(u_{R}, v_{R}\right)$ and $Q=\left(u_{Q}, v_{Q}\right)$ where $v_{P}<v_{R}<v_{Q} . P$ and $Q$ are stable constant solutions of (1.1), which guarantees the bistability, while $R$ is unstable.

\footnotetext{
* Received August 5, 1999; revised Sept 18, 2000.

†Department of Mathematics Faculty of Science Saitama University, 255 Shimo-Okubo, Urawa 338-8570, Japan (snii@rimath.saitama-u.ac.jp).
} 
Let $s=x+c t$ be the traveling coordinate. Then the system of equations for a traveling wave becomes:

$$
\left\{\begin{array}{l}
\dot{u}=\frac{1}{\varepsilon} u_{1} \\
\dot{u}_{1}=\frac{c \tau}{\varepsilon} u_{1}-\frac{1}{\varepsilon} f(u, v ; \theta, \gamma) \quad\left(\cdot=\frac{d}{d s}\right) \\
\dot{v}=v_{1} \\
\dot{v}_{1}=c v_{1}-g(u, v ; \theta, \gamma)
\end{array}\right.
$$

This system is simply written as:

$$
\dot{x}=\mathcal{F}(x ; \mu), \quad x=\left(\begin{array}{c}
u \\
u_{1} \\
v \\
v_{1}
\end{array}\right) \in \mathbb{R}^{4}, \quad \mu=\left(\begin{array}{c}
c \\
\theta \\
\gamma
\end{array}\right) \in \mathbb{R}^{3}
$$

This system has equilibria corresponding to $P$ and $Q$, which are denoted in the same notation by:

$$
P=\left(\begin{array}{c}
u_{P} \\
0 \\
v_{P} \\
0
\end{array}\right), \quad Q=\left(\begin{array}{c}
u_{Q} \\
0 \\
v_{Q} \\
0
\end{array}\right)
$$

The equilibrium $P$ (resp. $Q$ ) has a two-dimensional stable manifold $W^{s}(P)$ (resp. $\left.W^{s}(Q)\right)$ and a two-dimensional unstable manifold $W^{u}(P)$ (resp. $\left.W^{u}(Q)\right)$. In [13], a function $\Xi_{1}(\mu)$ (resp. $\Xi_{2}(\mu)$ ) is defined to analyze heteroclinic orbits from $P$ to $Q$ (resp. from $Q$ to $P$ ), which measures the separation of $W^{u}(P)$ and $W^{s}(Q)$ (resp. $W^{u}(Q)$ and $\left.W^{s}(P)\right)$ and satisfies the following property:

$\Xi_{1}=0$ if and only if there exists a heteroclinic orbit from $P$ to $Q$.

(resp. $\Xi_{2}=0$ if and only if there exists a heteroclinic orbit from $Q$ to $P$.)

It is, then, proven that $\Xi_{1}$ is expanded into the following Taylor expansion:

$$
\Xi_{1}(\mu)=F \theta+G c \gamma+H c^{3}+o\left(|\theta|+\left|c^{2} \gamma\right|+\left|c^{4}\right|\right)
$$

where $F, G$ and $H$ are positive if $\varepsilon$ and $\tau$ are sufficiently small. Moreover, $\Xi_{1}$ and $\Xi_{2}$ have the following relations:

$$
\Xi_{1}(c, \theta, \gamma)=-\Xi_{2}(-c, \theta, \gamma)
$$

and

$$
\Xi_{1}(c, \theta, \gamma)=\Xi_{2}(c,-\theta, \gamma)
$$

It follows from the information above that the set of zeros of $\Xi_{1}$ and $\Xi_{2}$ :

$$
M_{1}:=\left\{(c, \theta, \gamma) \in \mathbb{R}^{3} \mid \Xi_{1}(c, \theta, \gamma)=0\right\}, M_{2}:=\left\{(c, \theta, \gamma) \in \mathbb{R}^{3} \mid \Xi_{2}(c, \theta, \gamma)=0\right\}
$$

form cusp surfaces which are symmetric to each other. Especially, the bifurcation diagram in $c-\theta$ plane for fixed $\gamma$ has the following feature:

$\gamma_{*}>0 M_{1 \gamma_{*}}$ and $M_{2 \gamma_{*}}$ intersect transversally at $(c, \theta)=(0,0)$ 


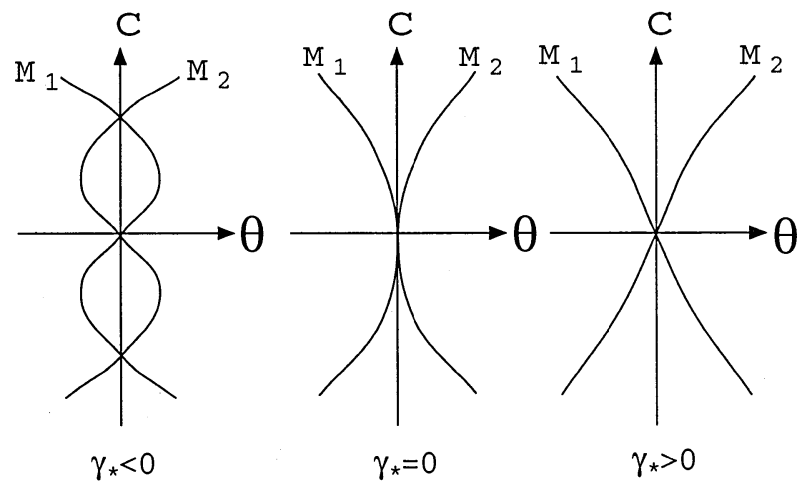

Figure 1 .

$\gamma_{*}=0$ they tangent at $(c, \theta)=(0,0)$

$\gamma_{*}<0$ they intersect transversally at three points including $(c, \theta)=(0,0)$

where $M_{i \gamma_{*}}=M_{i} \cap\left\{\gamma=\gamma_{*}\right\}$. (See figure 1.) As for the stability of waves corresponding to these heteroclinic orbits, the following is shown:

Let $\mu_{*} \in M_{1}$, then

$$
\frac{\partial}{\partial c} \Xi_{1}\left(\mu_{*}\right)>0 \quad\left(\frac{\partial}{\partial c} \Xi_{1}\left(\mu_{*}\right)<0\right)
$$

if and only if the traveling wave corresponding to the heteroclinic orbit is stable (resp. unstable) as a solution of the original system (1.1); the same applies for $\Xi_{2}$.

In [13], the existence of homoclinic orbits to $P$ and $Q$ bifurcating from the transversal intersection of $M_{1 \gamma_{*}}$ and $M_{2 \gamma_{*}}$ are obtained by applying homoclinic bifurcation theorem by Kokubu [12]. That is, when $M_{1 \gamma_{*}}$ and $M_{2 \gamma_{*}}$ intersect transversally i.e. $\quad \gamma_{*} \neq 0$, there are branches of curves $M_{P \gamma_{*}}=M_{P} \cap\left\{\gamma=\gamma_{*}\right\}$ and $M_{Q \gamma_{*}}=M_{Q} \cap\left\{\gamma=\gamma_{*}\right\}$ in $c-\theta$ plane corresponding to homoclinic orbits to $P$ and $Q$ which bifurcate from the intersection points of $M_{1 \gamma_{*}}$ and $M_{2 \gamma_{*}}$. (See figure 2.) The stability of traveling pulses corresponding to these homoclinic orbits is determined in [14]. The method used there also depends on the transversality.

At this stage, nothing had been proven concerning the existence of homoclinic orbits for $\gamma_{*}=0$. Later, an almost complete bifurcation diagram of homoclinic and heteroclinic orbits, and the stability and instability of the corresponding waves were obtained for piece-wise linear $f$ and $g$ by the singular perturbation technique (Ikeda, Ikeda and Mimura [8]). The existence of Hopf bifurcation of pulses was also shown. Still, these results exclude a small neighborhood of $(c, \theta, \gamma)=(0,0,0)$ in the parameter space, because of the lack of transversality.

The purpose of this paper is to investigate this type of degeneracy and to exhibit the bifurcation structure of pulses. Moreover, the existence of Hopf bifurcation of pulses is also proven.

2. The Theorems. In this section, the theorems of this paper shall be stated. First, a theorem concerning a heteroclinic bifurcation of a family of ordinary differential equations under certain condition is introduced. Pulse solutions for a system of reaction diffusion equations are obtained from an application of the theorem. Then, under additional assumptions of symmetry, a theorem concerning the Hopf bifurcation of the pulse solutions is stated. 


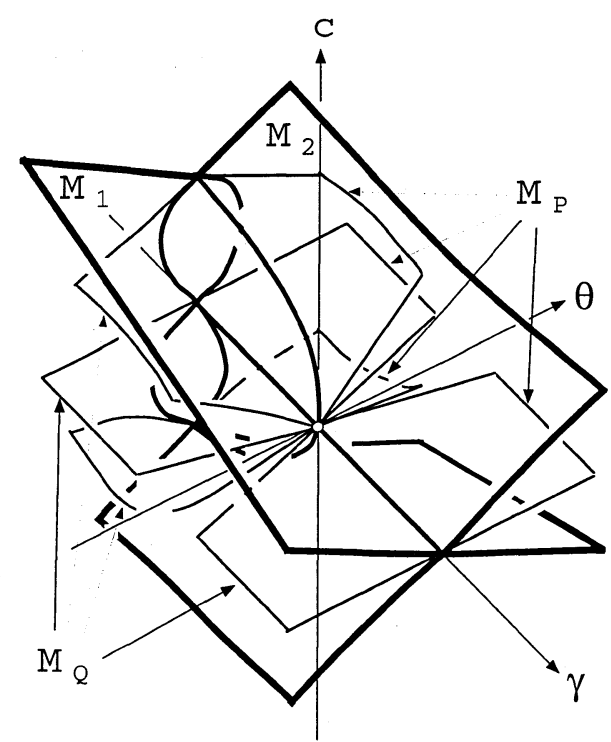

Figure 2.

The subject analysed first is a three parameter family of ordinary differential equations on $\mathbb{R}^{2 n}$ :

$$
\left\{\begin{array}{l}
\dot{x}=\mathfrak{X}\left(x, y ; \mu_{0}, \mu_{1}, \mu_{2}\right) \\
\dot{y}=\mathfrak{Y}\left(x, y ; \mu_{0}, \mu_{1}, \mu_{2}\right)
\end{array}\right.
$$

where $x, y \in \mathbb{R}^{n}$ and $\left(\mu_{0}, \mu_{1}, \mu_{2}\right) \in \mathbb{R}^{3}$.

In order to formulate the problem, this system is assumed to satisfy several hypotheses.

HypothesIS 1. The system (1.1) possesses the following symmetry:

$$
-\mathfrak{X}\left(y, x ;-\mu_{0}, \mu_{1}, \mu_{2}\right)=\mathfrak{Y}\left(x, y ; \mu_{0}, \mu_{1}, \mu_{2}\right)
$$

Hypothesis 2. The origin $O$ is a hyperbolic equilibrium of the system and there are other hyperbolic equilibria $\mathrm{O}_{-}$and $\mathrm{O}_{+}$. $\mathrm{O}_{-}$corresponds to $\mathrm{O}_{+}$under the symmetry in Hypothesis 1. $O\left(O_{ \pm}\right)$has an $n$-dimensional stable manifold $W^{s}(O)$ (resp. $W^{s}\left(O_{ \pm}\right)$) and an $n$-dimensional unstable manifold $W^{u}(O)$ (resp. $\left.W^{u}\left(O_{ \pm}\right)\right)$.

For the sake of simplicity, the system (2.1) is assumed to be linear around the origin $O$. Moreover, it is written in the following form for some small neighborhood around the origin:

$$
\left(\begin{array}{l}
\dot{x}_{1} \\
\dot{\bar{x}} \\
\dot{y}_{1} \\
\dot{\bar{y}}
\end{array}\right)=\left(\begin{array}{cccc}
-\lambda\left(\mu_{0}, \mu_{1}, \mu_{2}\right) & 0 & 0 & 0 \\
0 & -A\left(\mu_{0}, \mu_{1}, \mu_{2}\right) & 0 & 0 \\
0 & 0 & \lambda\left(-\mu_{0}, \mu_{1}, \mu_{2}\right) & 0 \\
0 & 0 & 0 & A\left(-\mu_{0}, \mu_{1}, \mu_{2}\right)
\end{array}\right)\left(\begin{array}{l}
x_{1} \\
\bar{x} \\
y_{1} \\
\bar{y}
\end{array}\right)
$$

where $\left(x_{1}, \bar{x}\right),\left(y_{1}, \bar{y}\right) \in \mathbb{R} \times \mathbb{R}^{n-1}, \lambda\left(\mu_{0}, \mu_{1}, \mu_{2}\right)>0$ and $A\left(\mu_{0}, \mu_{1}, \mu_{2}\right)$ is a $(n-1) \times$ $(n-1)$ matrix. 
HYPOTHESIS 3. $\frac{\partial \lambda}{\partial \mu_{0}}\left(0, \mu_{1}, \mu_{2}\right)<0$.

HYPOTHESIS 4. There is a positive constant $\nu>\lambda\left(\mu_{0}, \mu_{1}, \mu_{2}\right)$ satisfying the following property:

$$
\left\|e^{-A\left(\mu_{0}, \mu_{1}, \mu_{2}\right) t}\right\|<e^{-\nu t} \quad(t>0)
$$

As the subject of interest is the heteroclinic bifurcation of this system, the original heteroclinic solutions which undergo the bifurcation are assumed to exist.

Hypothesis 5. When $\left(\mu_{0}, \mu_{1}, \mu_{2}\right)=(0,0,0)(2.1)$ possesses a heteroclinic solution, which is unique up to translation in $t$

$$
h_{1}(t)=\left(h_{1}^{x}(t), h_{1}^{y}(t)\right) \text { satisfying } \lim _{t \rightarrow-\infty} h_{1}(t)=O_{-} \text {and } \lim _{t \rightarrow+\infty} h_{1}(t)=O .
$$

If $h_{2}(t):=\left(h_{1}^{y}(-t), h_{1}^{x}(-t)\right)$, then the symmetry in Hypothesis 1 implies that $h_{2}(t)$ is a heteroclinic solution satisfying $\lim _{t \rightarrow-\infty} h_{1}(t)=O$ and $\lim _{t \rightarrow+\infty} h_{1}(t)=O_{+}$.

These heteroclinic solutions are assumed to be of generic type i.e. they satisfy the following hypotheses.

HYPOTHESIS 6.

$$
\lim _{t \rightarrow+\infty} \frac{\dot{h}_{1}(t)}{\left|\dot{h}_{1}(t)\right|}=(-1,0,0,0)
$$

By the symmetry, the following also holds:

$$
\lim _{t \rightarrow-\infty} \frac{\dot{h}_{2}(t)}{\left|\dot{h}_{2}(t)\right|}=(0,0,1,0)
$$

Let $\Sigma_{i n}=\left\{x_{1}=\delta\right\}$ and $\Sigma_{o u t}=\left\{y_{1}=\delta\right\}$ be local sections which are transversal to the heteroclinic orbits and $\delta>0$ be so small that $\Sigma_{\text {in }}$ and $\Sigma_{\text {out }}$ are in the neighborhood of the origin where the linearity assumption (2.2) holds. (See figure 3.) In what follows, $\delta=1$ is assumed through a scalar change of the coordinates, and $\left(\bar{x}, y_{1}, \bar{y}\right)$ and $\left(x_{1}, \bar{x}, \bar{y}\right)$ are used as the coordinates on $\Sigma_{i n}$ and $\Sigma_{o u t}$.

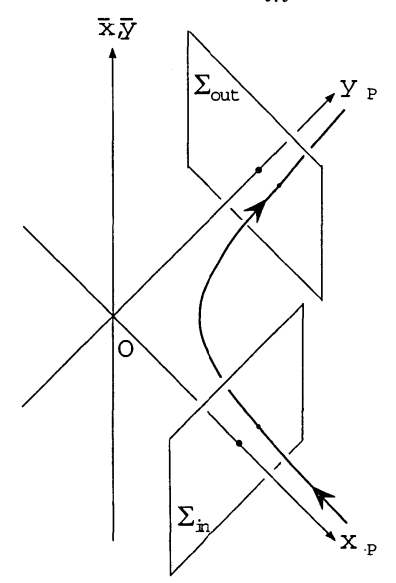

Figure 3 . 
Hypothesis 7. $W^{u}\left(O_{-}\right) \cap \Sigma_{i n}$ is expressed as the graph of a smooth function from $\bar{y}$ to $\left(\bar{x}, y_{1}\right)$ (see figure 4$)$ :

$$
\bar{x}=\xi\left(\bar{y} ; \mu_{0}, \mu_{1}, \mu_{2}\right), \quad y_{1}=\eta_{1}\left(\bar{y} ; \mu_{0}, \mu_{1}, \mu_{2}\right)
$$

By the symmetry in Hypothesis 1, this implies that $W^{s}\left(O_{+}\right) \cap \Sigma_{\text {out }}$ is expressed as the graph of a smooth function from $\bar{x}$ to $\left(x_{1}, \bar{y}\right)$ :

$$
x_{1}=\eta_{1}\left(\bar{x} ;-\mu_{0}, \mu_{1}, \mu_{2}\right), \quad \bar{y}=\xi\left(\bar{x} ;-\mu_{0}, \mu_{1}, \mu_{2}\right)
$$

The next hypothesis represents the degeneracy which is under investigation.

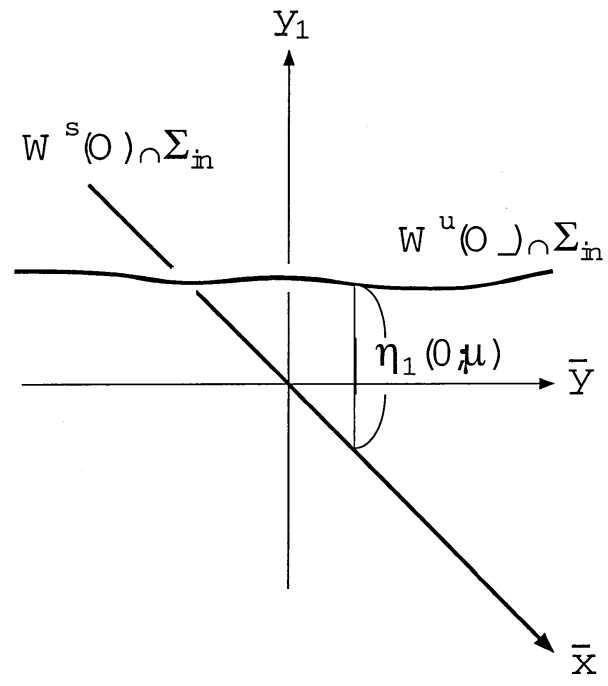

Figure 4 .

Hypothesis 8. The function $\eta_{1}$ has the following symmetry:

$$
\eta_{1}\left(0 ; \mu_{0}, \mu_{1}, \mu_{2}\right)=-\eta_{1}\left(0 ;-\mu_{0},-\mu_{1}, \mu_{2}\right)
$$

and is expanded into the following Taylor expansion:

$$
\eta_{1}\left(0 ; \mu_{0}, \mu_{1}, \mu_{2}\right)=\mu_{1}+\mu_{0} \mu_{2}+\mu_{0}^{3}+o\left(\left|\mu_{1}\right|+\left|\mu_{0}^{2} \mu_{2}\right|+\left|\mu_{0}^{4}\right|\right)
$$
[13].

REMARK 2.1. $\eta_{1}\left(0 ; \mu_{0}, \mu_{1}, \mu_{2}\right)$ corresponds to the separation function in [12] and

Let $M_{-}$and $M_{+}$be the parameter sets on which the heteroclinic solution from $O_{-}$to $O$ and from $O$ to $O_{+}$exists:

$$
\begin{gathered}
M_{-}:=\left\{\left(\mu_{0}, \mu_{1}, \mu_{2}\right) \mid \eta_{1}\left(0 ; \mu_{0}, \mu_{1}, \mu_{2}\right)=0\right\} \\
M_{+}:=\left\{\left(\mu_{0}, \mu_{1}, \mu_{2}\right) \mid \eta_{1}\left(0 ;-\mu_{0}, \mu_{1}, \mu_{2}\right)=0\right\}
\end{gathered}
$$

and let $\mathcal{P}:=M_{-} \cap M_{+}$be the parameter set on which both heteroclinic solutions coexist. Hypothesis 8 implies that $M_{-}$and $M_{+}$form cusp surfaces which are expressed 
by $\mu_{1}=c\left(\mu_{0}, \mu_{2}\right)$ and $\mu_{1}=c\left(-\mu_{0}, \mu_{2}\right)$, and that $\mathcal{P}$ consists of the line $\left\{\mu_{0}=\mu_{1}=0\right\}$ and the graphs of symmetric square-root-like functions $\left\{\mu_{0}=p\left(\mu_{2}\right), \mu_{2} \leq 0, \mu_{1}=\right.$ $0\} \cup\left\{\mu_{0}=-p\left(\mu_{2}\right), \mu_{2} \leq 0, \mu_{1}=0\right\}$ with $p\left(\mu_{2}\right) \geq 0$ and $p(0)=0$.

Under the hypotheses above, the following heteroclinic bifurcations occur.

THEOREM 1. Under Hypothesis 1 to Hypothesis 8, there exists a heteroclinic solution from $O_{-}$to $O_{+}$for $\left(0, \mu_{1}, \mu_{2}\right)$ with $\mu_{1}>0$. Furthermore, for $\mu_{2}<0$ with sufficiently small $\left|\mu_{2}\right|$, there exists a function $\phi\left(\mu_{0}, \mu_{2}\right)$ for $-p\left(\mu_{2}\right)<\mu_{0}<p\left(\mu_{2}\right)$ such that there exists a heteroclinic solution from $O_{-}$to $O_{+}$for $\left(\mu_{0}, \mu_{1}, \mu_{2}\right)=\left(\mu_{0}, \phi\left(\mu_{0}, \mu_{2}\right), \mu_{2}\right) . \phi\left(\mu_{0}, \mu_{2}\right)$ satisfies $\eta_{1}\left(0 ; \pm \mu_{0}, \phi\left(\mu_{0}, \mu_{2}\right), \mu_{2}\right)>0$ and $\lim _{\mu_{0} \rightarrow \pm p\left(\mu_{2}\right)} \phi\left(\mu_{0}, \mu_{2}\right)=0$. $\phi\left(0, \mu_{2}\right)$ converges to 0 as $\mu_{2} \uparrow 0$ like $\mu_{2}=\mu_{1} \log \mu_{1}$. (See figure 5.)

REMARK 2.2. The branches of the heteroclinic orbit is unique when $\mu_{2} \neq 0$ and $\mu_{1}$ is near 0 by the results of Kokubu [12].

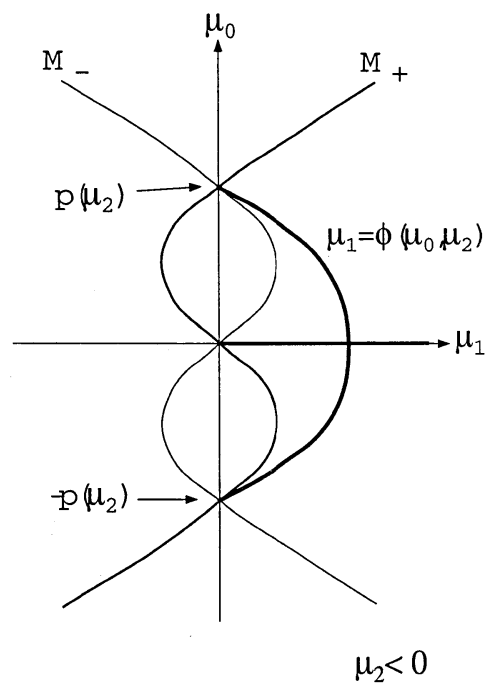

Figure 5 .

When this theorem is applied to the problem of traveling waves, the theorem concerning existence of pulse solutions is obtained. The subject here is the following system of reaction diffusion equations:

$$
u_{t}=B u_{x x}+f(u ; \mu)
$$

where $x \in \mathbb{R}, t>0$ and $u \in \mathbb{R}^{n} . \quad B$ is an $n \times n$ positive diagonal matrix and $f$ is a smooth function of $u$ depending on parameters $\mu=\left(\mu_{1}, \mu_{2}\right) \in \mathbb{R}^{2}$. This equation is considered on the function space $B U\left(\mathbb{R}, \mathbb{R}^{n}\right):=\left\{u: \mathbb{R} \rightarrow \mathbb{R}^{n} \mid u\right.$ is bounded and uniformly continuous $\}$. The basic hypothesis for this system is the following bistability

Hypothesis 9 . The non-linearity $f$ has two zeros $P$ and $Q$, and steady state constant solutions $u \equiv P$ and $u \equiv Q$ are both stable. 
Put $s=x+c t$ and $(2.3)$ in $(s, t)$ coordinate becomes:

$$
u_{t}=B u_{s s}-c u_{s}+f(u ; \mu)
$$

Then a traveling wave solution $u(x, t)$ is a stationary solution $u(x, t)=u(s)$ of $(2.4)$ for which $\lim _{s \rightarrow \pm \infty} u(s)$ exists and equals either $P$ or $Q$. That is, if the equation:

$$
B u_{s s}-c u_{s}+f(u ; \mu)=0
$$

possesses a solution $u(s)$ with $\lim _{s \rightarrow \pm \infty} u(s)=P$ or $Q$, then $u(x+c t)$ in $(x, t)$ coordinates becomes a traveling wave solution of the system (2.3). Notice that a traveling wave is called a traveling pulse if both of the limits of $\lim _{s \rightarrow \pm \infty} u(s)$ are equal to $P$ or both are equal to $Q$, and is called a standing wave (a standing pulse) if $c=0$.

The problem above is regarded as the existence problem of a homoclinic or heteroclinic solution. The second order ordinary differential equation (2.5) is rewritten as a first order system:

$$
\left\{\begin{array}{l}
\dot{u}=v \\
\dot{v}=c B^{-1} v-B^{-1} f\left(u ; \mu_{1}, \mu_{2}\right) \quad\left(\cdot=\frac{d}{d s}\right)
\end{array}\right.
$$

Under Hypothesis 9, the system (2.6) has two hyperbolic equilibria $(u, v)=(P, 0)$ and $(u, v)=(Q, 0)$. By abuse of notation, these equilibria are also denoted as $P$ and $Q$. Then $\left(u, u^{\prime}\right)$ is a homoclinic or heteroclinic solution to $P$ and/or $Q$ of (2.6) if and only if $u$ is a solution of (2.5) with $\lim _{s \rightarrow \pm \infty} u(s)=P$ or $Q$.

In what follows, $f$ is assumed to be linear around $P$ corresponding to the assumption (2.2). Then, by letting $O=P, O_{-}=O_{+}=Q$ and $\left(\mu_{0}, \mu_{1}, \mu_{2}\right)=\left(c, \mu_{1}, \mu_{2}\right)$, Hypothesis 1 and Hypothesis 2 are automatically satisfied after a suitable change of coordinates. This system is, then, expressed as in (2.2).

In this context, theorem 1 is interpreted as follows.

THEOREM 2. If (2.6) has heteroclinic solutions which correspond to traveling waves of (2.3) and which satisfy the above hypotheses, then the system possesses a homoclinic solution to $Q$ for $c=0$ and $\mu_{1}>0$ which corresponds to a standing pulse of (2.3). Furthermore, the system has a homoclinic solution to $Q$ for $\mu_{1}=\phi\left(c, \mu_{2}\right)$ for $\mu_{2}<0$ which corresponds to a traveling pulse with speed $c$.

REMARK 2.3. From this point of view, the bifurcation point $\left(c, \mu_{1}, \mu_{2}\right)=$ $\left(0, \phi\left(0, \mu_{2}\right), \mu_{2}\right)$ of a traveling pulse from a standing pulse is the pitchfork bifurcation point of the standing pulse.

In the context of traveling waves, this degeneracy generates a richer structure. In fact, it is shown that the standing wave undergoes Hopf bifurcation under additional hypotheses.

The first hypothesis is a symmetry of the system (2.3).

Hypothesis 10. The non-linearity $f$ is odd symmetric with respect to $u$ and $\mu_{1}$ :

$$
f\left(-u ;-\mu_{1}, \mu_{2}\right)=-f\left(u ; \mu_{1}, \mu_{2}\right)
$$

If $P$ and $Q$ correspond to each other under this symmetry, then $Q=-P$ for $\mu_{1}=0$.

The next hypothesis concerns the linear stability of traveling waves. 
Let $u(s)$ be a traveling waves of (2.3). Consider the linearization of the system (2.4) along the wave:

$$
p_{t}=L p:=B p_{s s}-c p_{s}+D f(u(s) ; \mu) p
$$

Under Hypothesis 9, there is a negative constant $\sigma_{0}$ such that the essential spectrum $\sigma_{e}(L)$ of $L$ on the function space $B U\left(\mathbb{R}, \mathbb{R}^{n}\right)$ lies in the region $\Re z<\sigma_{0}$. Moreover the origin is an eigenvalue of $L$ with the eigenfunction $u_{s}(s)$ which corresponds to spatial translation of the wave. The wave is said to be linearly stable if there is no eigenvalue of $L$ with non-negative real part and linearly unstable if there is an eigenvalue of $L$ with positive real part.

REMARK 2.4. In the context of reaction diffusion systems, it is well known that linear stability of a wave implies non-linear stability. See Henry [7], for example.

Let $u_{-}\left(s ; c, \mu_{1}, \mu_{2}\right)$ be the traveling wave satisfying $\lim _{s \rightarrow-\infty} u_{-}(s)=Q$ and $\lim _{s \rightarrow+\infty} u_{-}(s)=P$, which exists for $\left(c, \mu_{1}, \mu_{2}\right) \in M_{-}$, and let $u_{+}\left(s ; c, \mu_{1}, \mu_{2}\right)$ be the wave satisfying $\lim _{s \rightarrow-\infty} u_{+}(s)=P$ and $\lim _{s \rightarrow+\infty} u_{+}(s)=Q$, which exists for $\left(c, \mu_{1}, \mu_{2}\right) \in M_{+}$.

HyPOTHESIS 11. Let $L_{-}$be the linearized operator corresponding to the traveling wave $u_{-}\left(s ; c, \mu_{1}, \mu_{2}\right)$. Then there is a negative constant $\sigma_{1}$ so that there are only two eigenvalues of $L_{-}$with real part greater than $\sigma_{1}$. Moreover, one of these is negative (positive) if and only if

$$
\frac{\partial \eta_{1}}{\partial c}\left(0 ; c, \mu_{1}, \mu_{2}\right)>0 \quad\left(\text { resp. } \frac{\partial \eta_{1}}{\partial c}\left(0 ; c, \mu_{1}, \mu_{2}\right)<0\right)
$$

(The other one is at the origin.) Similarly, let $L_{+}$be the linearized operator corresponding to the traveling wave $u_{+}\left(s ; c, \mu_{1}, \mu_{2}\right)$. Then there are only two eigenvalues of $L_{+}$with real part greater than $\mu_{1}$, one of which is negative (positive) if and only if

$$
\frac{\partial \eta_{1}}{\partial c}\left(0 ;-c, \mu_{1}, \mu_{2}\right)<0 \quad\left(\text { resp. } \frac{\partial \eta_{1}}{\partial c}\left(0 ; c, \mu_{1}, \mu_{2}\right)>0\right)
$$

Under these hypotheses, the standing pulse, the existence of which is proven in theorem 2, undergoes Hopf bifurcation.

THEOREM 3. Under the additional Hypotheses 10 and 11, there is a curve $\mu_{2}=$ $\mu_{2}\left(\mu_{1}\right)$ for $\mu_{1}>0$ such that the linearization along the standing pulse possesses a pair of pure imaginary eigenvalues for $\left(\mu_{1}, \mu_{2}\right)=\left(\mu_{1}, \mu_{2}\left(\mu_{1}\right)\right)$, and $\mu_{2}\left(\mu_{1}\right)$ satisfies $\lim _{\mu_{1} \downarrow 0} \mu_{2}\left(\mu_{1}\right)=0$.

3. Heteroclinic bifurcations. In this section, theorem 1 and 2 shall be proven. To begin with, the bifurcation equation is formulated.

Proposition 3.1. The system (2.1) possesses a heteroclinic solution from $O_{-}$ to $\mathrm{O}_{+}$if and only if the following system of equations has a solution $(\bar{x}, \bar{y})$ :

$$
\left\{\begin{array}{l}
\left\{\eta_{1}\left(\bar{y} ; \mu_{0}, \mu_{1}, \mu_{2}\right)\right\}^{\frac{\lambda\left(\mu_{0}, \mu_{1}, \mu_{2}\right)}{\lambda\left(\mu_{0}, \mu_{1}, \mu_{2}\right)}}=\eta_{1}\left(\bar{x} ;-\mu_{0}, \mu_{1}, \mu_{2}\right) \\
\bar{x}=\exp \left\{\frac{1}{\lambda\left(-\mu_{0}, \mu_{1}, \mu_{2}\right)} \log \eta_{1}\left(\bar{y} ; \mu_{0}, \mu_{1}, \mu_{2}\right) A\left(\mu_{0}, \mu_{1}, \mu_{2}\right)\right\} \xi\left(\bar{y} ; \mu_{0}, \mu_{1}, \mu_{2}\right) \\
\bar{y}=\exp \left\{\frac{1}{\lambda\left(-\mu_{0}, \mu_{1}, \mu_{2}\right)} \log \eta_{1}\left(\bar{y} ; \mu_{0}, \mu_{1}, \mu_{2}\right) A\left(-\mu_{0}, \mu_{1}, \mu_{2}\right)\right\} \xi\left(\bar{x} ;-\mu_{0}, \mu_{1}, \mu_{2}\right)
\end{array}\right.
$$


Proof. By Hypothesis 7, a point in $W^{u}\left(O_{-}\right) \cap \Sigma_{i n}$ is expressed as:

$$
\left(\bar{x}, y_{1}, \bar{y}\right)=\left(\xi\left(\bar{y} ; \mu_{0}, \mu_{1}, \mu_{2}\right), \eta_{1}\left(\bar{y}, \mu_{0}, \mu_{1}, \mu_{2}\right), \bar{y}\right)
$$

and a point in $W^{s}\left(O_{+}\right) \cap \Sigma_{\text {out }}$ as:

$$
\left(x_{1}, \bar{x}, \bar{y}\right)=\left(\eta_{1}\left(\bar{x} ;-\mu_{0}, \mu_{1}, \mu_{2}\right), \bar{x}, \xi\left(\bar{x} ;-\mu_{0}, \mu_{1}, \mu_{2}\right)\right)
$$

Then, the condition that the orbit of a point in $W^{u}\left(O_{-}\right) \cap \Sigma_{i n}$ under the flow of (2.2) hits a point in $W^{s}\left(O_{+}\right) \cap \Sigma_{\text {out }}$ is expressed as (3.1).

Then, the first half of theorem 1 is easily proven.

Proposition 3.2. For $\mu_{0}=0$ and $\mu_{1}>0$, the system (2.1) possesses a heteroclinic solution from $O_{-}$to $O_{+}$if $\left|\mu_{1}\right|$ and $\left|\mu_{2}\right|$ are sufficiently small.

Proof. If $\bar{x}=\bar{y}$, then the first equation of (3.1) is automatically satisfied and the second equation coincides with the third equation. Let $F: \mathbb{R}^{n-1} \times \mathbb{R}^{2} \rightarrow \mathbb{R}^{n-1}$ be defined as:

$$
F\left(\bar{y} ; \mu_{1}, \mu_{2}\right):=\left\{\begin{array}{cc}
\bar{y}-\exp \left\{\frac{1}{\lambda\left(0, \mu_{1}, \mu_{2}\right)} \log \left|\eta_{1}\left(\bar{y} ; 0, \mu_{1}, \mu_{2}\right)\right| A\left(0, \mu_{1}, \mu_{2}\right)\right\} & \xi\left(\bar{y} ; 0, \mu_{1}, \mu_{2}\right) \\
\left(\eta_{1}\left(\bar{y} ; 0, \mu_{1}, \mu_{2}\right)\right. & \neq 0) \\
\bar{y} & \left(\eta_{1}\left(\bar{y} ; 0, \mu_{1}, \mu_{2}\right)=0\right)
\end{array}\right.
$$

Then, $F\left(0 ; 0, \mu_{2}\right)=0$ and $F$ is of class $C^{1}$ by Hypothesis 4 . Furthermore, $\frac{\partial}{\partial \bar{y}} F\left(0 ; 0, \mu_{2}\right)$ $=I$ and thus there is a function $\bar{y}=\bar{y}\left(\mu_{1}, \mu_{2}\right)$ satisfying $F\left(\bar{y}\left(\mu_{1}, \mu_{2}\right) ; \mu_{1}, \mu_{2}\right)=0$ for small $\left|\mu_{1}\right|$. A similar calculation yields $\frac{\partial \bar{y}}{\partial \mu_{1}}=\frac{\partial \bar{y}}{\partial \mu_{2}}=0$ at $\mu_{1}=0$ which means $\eta_{1}\left(\bar{y} ; 0, \mu_{1}, \mu_{2}\right)>0$ for positive small $\mu_{1}$ by Hypothesis 8 . Therefore, (3.1) is satisfied by $\bar{x}=\bar{y}=\bar{y}\left(\mu_{1}, \mu_{2}\right)$ for sufficiently small positive $\mu_{1}$.

To tackle the case $\mu_{0} \neq 0$, the second and the third equation are solved first.

LEMma 3.1. Let $G: \mathbb{R}^{n-1} \times \mathbb{R}^{n-1} \times \mathbb{R}^{3} \rightarrow \mathbb{R}^{n-1} \times \mathbb{R}^{n-1}$ be defined as:

$$
\begin{aligned}
& G\left(\bar{x}, \bar{y} ; \mu_{0}, \mu_{1}, \mu_{2}\right) \\
& \quad:=\left(\begin{array}{c}
\bar{x}-\exp \left\{\frac{1}{\lambda\left(-\mu_{0}, \mu_{1}, \mu_{2}\right)} \log \left|\eta_{1}\left(\bar{y} ; \mu_{0}, \mu_{1}, \mu_{2}\right)\right| A\left(\mu_{0}, \mu_{1}, \mu_{2}\right)\right\} \xi\left(\bar{y} ; \mu_{0}, \mu_{1}, \mu_{2}\right) \\
\bar{y}-\exp \left\{\frac{1}{\lambda\left(-\mu_{0}, \mu_{1}, \mu_{2}\right)} \log \left|\eta_{1}\left(\bar{y} ; \mu_{0}, \mu_{1}, \mu_{2}\right)\right| A\left(-\mu_{0}, \mu_{1}, \mu_{2}\right)\right\} \xi\left(\bar{x} ;-\mu_{0}, \mu_{1}, \mu_{2}\right)
\end{array}\right)
\end{aligned}
$$

if $\eta_{1}\left(\bar{y} ; \mu_{0}, \mu_{1}, \mu_{2}\right) \neq 0$ and

$$
G\left(\bar{x}, \bar{y} ; \mu_{0}, \mu_{1}, \mu_{2}\right):=(\bar{x}, \bar{y})
$$

if $\eta_{1}\left(\bar{y} ; \mu_{0}, \mu_{1}, \mu_{2}\right)=0$, then there is a function $X\left(\mu_{0}, \mu_{1}, \mu_{2}\right)$ and $Y\left(\mu_{0}, \mu_{1}, \mu_{2}\right)$ with $X(0,0,0)=Y(0,0,0)=0$ which solves the equation

$$
G\left(X\left(\mu_{0}, \mu_{1}, \mu_{2}\right), Y\left(\mu_{0}, \mu_{1}, \mu_{2}\right), \mu_{0}, \mu_{1}, \mu_{2}\right)=0
$$

for sufficiently small $\left|\mu_{0}\right|,\left|\mu_{1}\right|$ and $\left|\mu_{2}\right|$.

Proof. This is also a consequence of Hypothesis 4 and the implicit function theorem. 
Moreover, the following is easily seen.

LEMMA 3.2.

(1) $X\left(\mu_{0}, \mu_{1}, \mu_{2}\right)=Y\left(\mu_{0}, \mu_{1}, \mu_{2}\right)=0$ if $\left(\mu_{0}, \mu_{1}, \mu_{2}\right) \in M_{-}$.

(2) $\frac{\partial X}{\partial \mu_{0}}=\frac{\partial X}{\partial \mu_{1}}=\frac{\partial X}{\partial \mu_{2}}=\frac{\partial Y}{\partial \mu_{0}}=\frac{\partial Y}{\partial \mu_{1}}=\frac{\partial Y}{\partial \mu_{2}}=0$ at $\left(\mu_{0}, \mu_{1}\right)=(0,0)$.

This lemma implies the following.

LEMMA 3.3. $\eta_{1}\left(Y\left(\mu_{0}, \mu_{1}, \mu_{2}\right) ; \mu_{0}, \mu_{1}, \mu_{2}\right)>0$ holds if $\left|\mu_{0}\right|$ and $\left|\mu_{1}\right|$ are sufficiently small and $\mu_{1}$ satisfies $c\left(\mu_{0}, \mu_{2}\right)<\mu_{1}$.

In what follows, $\mu_{0} \geq 0$ is assumed because the results for the other case automatically follow from the symmetry. (Hypothesis 1 )

In order to find a solution of the first equation of (3.1), the following function is considered:

$$
H\left(\mu_{0}, \mu_{1}, \mu_{2}\right):=\left\{\begin{array}{cc}
\frac{1}{\mu_{0}}\left\{\eta_{1}\left(Y\left(\mu_{0}, \mu_{1}, \mu_{2}\right), \mu_{0}, \mu_{1}, \mu_{2}\right)^{\lambda\left(\mu_{0}, \mu_{1}, \mu_{2}\right)}\right. & \\
\left.-\eta_{1}\left(X\left(\mu_{0}, \mu_{1}, \mu_{2}\right),-\mu_{0}, \mu_{1}, \mu_{2}\right)^{\lambda\left(-\mu_{0}, \mu_{1}, \mu_{2}\right)}\right\} & \left(\mu_{0}>0\right) \\
\frac{\partial}{\partial \mu_{0}} \mid \begin{array}{c}
\mid \eta_{1}\left(Y\left(\mu_{0}, \mu_{1}, \mu_{2}\right), \mu_{0}, \mu_{1}, \mu_{2}\right)^{\lambda\left(\mu_{0}, \mu_{1}, \mu_{2}\right)} \\
\mu_{0}=0 \\
\left.-\eta_{1}\left(X\left(\mu_{0}, \mu_{1}, \mu_{2}\right),-\mu_{0}, \mu_{1}, \mu_{2}\right)^{\lambda\left(-\mu_{0}, \mu_{1}, \mu_{2}\right)}\right\}
\end{array} & \left(\mu_{0}=0\right)
\end{array}\right.
$$

Then for $\mu_{0}>0, H\left(\mu_{0}, \mu_{1}, \mu_{2}\right)=0$ if and only if the system (2.1) possesses a heteroclinic orbit from $\mathrm{O}_{-}$to $\mathrm{O}_{+}$.

The next proposition proves the second half of the theorem.

Proposition 3.3. There exists $\mu_{2 *}<0$ such that the following holds. For each $\mu_{2 *}<\mu_{2}<0$ and $0 \leq \mu_{0}<p\left(\mu_{2}\right)$ there exists $\mu_{1}$ with $c\left(\mu_{0}, \mu_{2}\right) \leq \mu_{1}$ satisfying $H\left(\mu_{0}, \mu_{1}, \mu_{2}\right)=0$.

Proof. First, there are constants $C_{1}$ and $C_{2}$ and a function $C_{3}\left(\mu_{1}, \mu_{2}\right)>0$ such that $H\left(\mu_{0}, \mu_{1}, \mu_{2}\right)$ is defined for $0<\mu_{1}<C_{1},\left|\mu_{2}\right|<C_{2}$ and $\left|\mu_{0}\right|<C_{3}\left(\mu_{1}, \mu_{2}\right)$ by the proof of the proposition 3.2 and the continuity of $\eta_{1}\left(Y\left(\mu_{0}, \mu_{1}, \mu_{2}\right) ; \mu_{0}, \mu_{1}, \mu_{2}\right)$ and $\eta_{1}\left(X\left(\mu_{0}, \mu_{1}, \mu_{2}\right) ;-\mu_{0}, \mu_{1}, \mu_{2}\right)$. Moreover, lemma 3.2 implies that there are constants $C_{4}$ and $C_{5}$ such that $\eta_{1}\left(Y\left(\mu_{0}, \mu_{1}, \mu_{2}\right) ; \mu_{0}, \mu_{1}, \mu_{2}\right)>0$ for $\left|\mu_{0}\right|<C_{4},\left|\mu_{2}\right|<C_{2}$ and $c\left(\mu_{0}, \mu_{2}\right)<\mu_{1}<C_{5}$. Therefore there is a function $C_{6}\left(\mu_{0}, \mu_{2}\right)$ such that $H$ is defined for $c\left(\mu_{0}, \mu_{2}\right)<\mu_{1}<C_{6}\left(\mu_{0}, \mu_{2}\right)$ if $0<\mu_{0}<p\left(\mu_{2}\right)$ and $-C_{2}<\mu_{2}<0$ because $\eta_{1}\left(X\left(\mu_{0}, \mu_{1}, \mu_{2}\right) ;-\mu_{0}, \mu_{1}, \mu_{2}\right)>0$ for $\left(\mu_{0}, \mu_{1}, \mu_{2}\right) \in M_{-}$.

$H\left(0, \mu_{1}, \mu_{2}\right)$ is explicitly expressed as:

$$
\begin{aligned}
& H\left(0, \mu_{1}, \mu_{2}\right) \\
= & \eta_{1}\left(Y\left(0, \mu_{1}, \mu_{2}\right), 0, \mu_{1}, \mu_{2}\right)^{\lambda\left(0, \mu_{1}, \mu_{2}\right)-1} \\
& \times\left[\lambda ( 0 , \mu _ { 1 } , \mu _ { 2 } ) \left\{\frac{\partial \eta_{1}}{\partial \bar{y}}\left(Y\left(0, \mu_{1}, \mu_{2}\right), 0, \mu_{1}, \mu_{2}\right) \frac{\partial Y}{\partial \mu_{0}}\left(0, \mu_{1}, \mu_{2}\right)\right.\right. \\
& \left.-\frac{\partial \eta_{1}}{\partial \bar{y}}\left(X\left(0, \mu_{1}, \mu_{2}\right), 0, \mu_{1}, \mu_{2}\right) \frac{\partial X}{\partial \mu_{0}}\left(0, \mu_{1}, \mu_{2}\right)+2 \frac{\partial \eta_{1}}{\partial \mu_{0}}\left(0, \mu_{1}, \mu_{2}\right)\right\} \\
& \left.+2 \frac{\partial \lambda}{\partial \mu_{0}}\left(0, \mu_{1}, \mu_{2}\right) \eta_{1}\left(Y\left(0, \mu_{1}, \mu_{2}\right), 0, \mu_{1}, \mu_{2}\right) \log \eta_{1}\left(Y\left(0, \mu_{1}, \mu_{2}\right), 0, \mu_{1}, \mu_{2}\right)\right]
\end{aligned}
$$


Because $\frac{\partial \lambda}{\partial \mu_{0}}\left(0, \mu_{1}, \mu_{2}\right)<0, H\left(0, \mu_{1}, \mu_{2}\right)$ behaves like $\mu_{2}-\mu_{1} \log \mu_{1}$. Therefore there is a constant $C_{7}$ and a function $C_{8}\left(\mu_{2}\right)$ with $C_{8}(0)=0$ such that $H$ is positive if $0 \leq \mu_{0}<C_{7},\left|\mu_{2}\right|<C_{2}$ and $C_{8}\left(\mu_{2}\right)<\mu_{1}$.

On the other hand, $H$ is negative if $\mu_{2}<0,0 \leq \mu_{0}<p\left(\mu_{2}\right)$ and $\mu_{1}=c\left(\mu_{0}, \mu_{2}\right)$ as $\eta_{1}\left(0 ; \mu_{0}, \mu_{1}, \mu_{2}\right)=0$ and $\eta_{1}\left(0 ;-\mu_{0}, \mu_{1}, \mu_{2}\right)>0$ there.

Take $\mu_{2 *}<0$ such that $\left|\mu_{2}\right|<C_{2}, C_{8}\left(\mu_{2}\right)<C_{5}$ and $p\left(\mu_{2}\right)<\min \left\{C_{4}, C_{7}\right\}$ holds for $\mu_{2 *}<\mu_{2}<0$. Then, for each $\mu_{2 *}<\mu_{2}<0$ and $0 \leq \mu_{0}<p\left(\mu_{2}\right)$, $\eta_{1}\left(Y\left(\mu_{0}, \mu_{1}, \mu_{2}\right) ; \mu_{0}, \mu_{1}, \mu_{2}\right)$ is positive for $c\left(\mu_{0}, \mu_{2}\right)<\mu_{1}<C_{5}$, and $H\left(\mu_{0}, \mu_{1}, \mu_{2}\right)$ is defined and positive for $C_{8}\left(\mu_{2}\right)<\mu_{1}<C_{5}$.

When $\left(\mu_{0}, \mu_{2}\right)$ is fixed in this region and $\mu_{1}$ is increased from $c\left(\mu_{0}, \mu_{2}\right)$ to $C_{5}$, either of the following happens:

(1) $H$ is defined for all $c\left(\mu_{0}, \mu_{2}\right) \leq \mu_{1} \leq C_{5}$ and $H<0$ for $\mu_{1}=c\left(\mu_{0}, \mu_{2}\right)$ and $H>0$ for $\mu_{1}=C_{5}$.

(2) There is some $c\left(\mu_{0}, \mu_{2}\right)<\mu_{1 *}<C_{5}$ such that $H$ is defined for $c\left(\mu_{0}, \mu_{2}\right)<$ $\mu_{1}<\mu_{1 *}$ and $H<0$ for $\mu_{1}=c\left(\mu_{0}, \mu_{2}\right)$, but $\eta_{1}\left(X\left(\mu_{0}, \mu_{1}, \mu_{2}\right) ;-\mu_{0}, \mu_{1}, \mu_{2}\right)$ becomes zero at $\mu_{1}=\mu_{1 *}$

The conclusion immediately follows by taking into account that $H\left(\mu_{1 *}\right)>0$ for the second case.

Proof of theorem 1. The theorem for $0 \leq \mu_{0}$ is proven in proposition 3.3. The conclusion for $\mu_{0}<0$ follows from the symmetry (Hypothesis 1 ). Asymptotic of the bifurcation curve follows from (3.2).

Proof of theorem 2. Recall the system of the traveling wave (2.6):

$$
\left\{\begin{array}{l}
\dot{u}=v \\
\dot{v}=c B^{-1} v-B^{-1} f\left(u ; \mu_{1}, \mu_{2}\right)
\end{array}\right.
$$

This system is simply written as

$$
\left(\begin{array}{c}
\dot{u} \\
\dot{v}
\end{array}\right)=F\left(u, v ; c, \mu_{1}, \mu_{2}\right)
$$

Let $D F\left(P, 0 ; c, \mu_{1}, \mu_{2}\right)$ be the linearization of $F$ around the equilibrium $P$. If $\lambda\left(c, \mu_{1}, \mu_{2}\right)$ is an eigenvalue and $V=(u, v)$ is a generalized eigenvector associated with $\lambda$, then the symmetry implies that $W=(u,-v)$ is an eigenvector of $D F\left(P, 0 ;-c, \mu_{1}, \mu_{2}\right)$ associated with the eigenvalue $-\lambda\left(c, \mu_{1}, \mu_{2}\right)$. Thus, if $V_{1}(c)=\left(u_{1}(c), v_{1}(c)\right), \ldots, V_{n}(c)$ $=\left(u_{n}(c), v_{n}(c)\right)$ are $n$ eigenvectors associated with eigenvalues $\lambda_{1}\left(c, \mu_{1}, \mu_{2}\right), \ldots$, $\lambda_{n}\left(c, \mu_{1}, \mu_{2}\right)$, then the other eigenvectors are obtained as $W_{1}(c)=\left(u_{1}(-c),-v_{1}(-c)\right)$, $\ldots, W_{n}(c)=\left(u_{n}(-c),-v_{n}(-c)\right)$ and belong to the eigenvalues $-\lambda_{1}\left(-c, \mu_{1}, \mu_{2}\right), \ldots$, $-\lambda_{n}\left(-c, \mu_{1}, \mu_{2}\right)$. Therefore, if $\left(V_{1}, \ldots, V_{n}, W_{1}, \ldots, W_{n}\right)$ are the basis of the coordinates, the system (2.6) possesses the symmetry of Hypothesis 1, and is expressed as (2.2). Hypothesis 2 also holds if Hypothesis 9 holds.

Then theorem 2 is obtained by applying theorem 1 .

4. Hopf bifurcations. In this section, theorem 3 shall be proven. In order to do this, a geometric interpretation of the eigenvalue problem is introduced, and then the proof of the theorem based on this interpretation is presented.

4.1. Geometric interpretation of the eigenvalue problem. Let $u(s)$ be a traveling wave of $(2.3)-i . e . \quad\left(u(s), u^{\prime}(s)\right)$ is a homoclinic/heteroclinic solution of 
(2.6) to/between $P$ or/and $Q$-and consider the eigenvalue problem associated with the linearization along the wave:

$$
L p=B p_{s s}-c p_{s}+D f(u(s) ; \mu) p=\Lambda p
$$

This system is also treated in the form of the first order system:

$$
\left\{\begin{array}{l}
\dot{p}=q \\
\dot{q}=c B^{-1} q+B^{-1}\left\{\Lambda-D f\left(u(s) ; \mu_{1}, \mu_{2}\right)\right\} p
\end{array}\right.
$$

This system is simply rewritten as:

$$
\dot{z}=\Gamma(u(s) ; \Lambda ; c, \mu) z
$$

Also consider the $n$-th exterior power of (4.2):

$$
\dot{z}^{(n)}=\Gamma^{(n)}(u(s) ; \Lambda ; c, \mu) z^{(n)}
$$

where $z^{(n)} \in \stackrel{n}{\wedge} \mathbb{C}^{2 n}$ and $\Gamma^{(n)}:=\Gamma \otimes I \otimes \cdots \otimes I+\cdots+\left.I \otimes \cdots \otimes I \otimes \Gamma\right|_{\wedge \mathbb{C}^{2 n}}$. Notice that if $z_{1}(s), \ldots, z_{n}(s)$ are solutions of $(4.2)$, then $z_{1}(s) \wedge \cdots \wedge z_{n}(s)$ is a solution of (4.3)

Under Hypothesis 9, there exists a negative constant $\sigma_{0}$ such that for $\Re \Lambda>\sigma_{0}$ the matrix $\Gamma_{ \pm}(\Lambda):=\lim _{s \rightarrow \pm \infty} \Gamma(u(s) ; \Lambda)$ has $n$ eigenvalues $\lambda_{1}^{ \pm}, \ldots, \lambda_{n}^{ \pm}$with negative real part and $n$ eigenvalues $\lambda_{n+1}^{ \pm}, \ldots, \lambda_{2 n}^{ \pm}$with positive real part. Moreover, $\Re \lambda_{i}<\lambda_{1}^{ \pm}<0$ for $2 \leq i \leq n$ and $0<\lambda_{n+1}^{ \pm}<\Re \lambda_{i}^{ \pm}$for $n+2 \leq i \leq 2 n$ holds under Hypothesis 4 . Then $\Gamma_{ \pm}^{(n)}(\Lambda):=\lim _{s \rightarrow \pm \infty} \Gamma^{(n)}(s ; \Lambda)$ has a simple eigenvalue $\lambda_{I}^{ \pm}=\sum_{i=n+1}^{2 n} \lambda_{i}^{ \pm}$having the largest real part among the eigenvalues and a simple eigenvalues $\lambda_{I I}^{ \pm}=\sum_{i=1}^{n} \lambda_{i}^{ \pm}$having the smallest real part. (See [1].) Let $z_{1 \pm}^{(n)}$ be the eigenvector associated with the eigenvalue $\lambda_{I}^{ \pm}$and $z_{2 \pm}^{(n)}$ be the eigenvector associated with the eigenvalue $\lambda_{I I}^{ \pm}$.

As the problem (4.1) is treated on the function space $B U\left(\mathbb{R}, \mathbb{R}^{n}\right), \Lambda$ is an eigenvalue of $L$ if and only if (4.1) has a bounded non-trivial solution. This is just to say that the system (4.2) has a non-trivial solution which converges to zero when $s \rightarrow \pm \infty$. This is reformulated in the following manner.

Let $W_{-}:=\left\{z(s) \mid\right.$ a solution of (4.2) satisfying $\left.\lim _{s \rightarrow-\infty} z(s)=0\right\}$ and $W_{-}\left(s_{0}\right):=$ $\left\{z\left(s_{0}\right) \mid z(s) \in W_{-}\right\}$. Similarly let $W_{+}:=\{z(s) \mid$ a solution of (4.2) satisfying $\left.\lim _{s \rightarrow+\infty} z(s)=0\right\}$ and $W_{+}\left(s_{0}\right):=\left\{z\left(s_{0}\right) \mid z(s) \in W_{+}\right\}$. Then $W_{ \pm}\left(s_{0}\right)$ is an $n-$ dimensional subspace of $\mathbb{C}^{2 n}$. The following is easily seen.

LEMMA 4.1. $\Lambda$ is an eigenvalue if and only if $W_{+}\left(s_{0}\right) \cap W_{-}\left(s_{0}\right) \neq\{0\}$.

This lemma is interpreted as follows. Let $z_{1}^{(n)}(s ; \Lambda)$ be a solution of (4.3) which converges to 0 as $s \rightarrow-\infty$ along the eigenspace $\operatorname{span}\left\{z_{1-}^{(n)}\right\}$ of $\Gamma_{-}^{(n)}$ and let $z_{2}^{(n)}(s ; \Lambda)$ be a solution of (4.3) which converges to 0 as $s \rightarrow+\infty$ along the eigenspace $\operatorname{span}\left\{z_{2+}^{(n)}\right\}$ of $\Gamma_{+}^{(n)} \cdot z_{1}^{(n)}$ and $z_{2}^{(n)}$ can be chosen such that they are analytic in $\Lambda$.

LEMMA 4.2 ([1]). $\Lambda$ is an eigenvalue if and only if $z_{1}^{(n)}(s ; \Lambda) \wedge z_{2}^{(n)}(s ; \Lambda)=0$ as an element in $\mathbb{C} \cong\left(\stackrel{n}{\wedge} \mathbb{C}^{2 n}\right) \wedge\left(\stackrel{n}{\wedge} \mathbb{C}^{2 n}\right)=\stackrel{2 n}{\wedge} \mathbb{C}^{2 n}$. 
LEMMA 4.3. $\Lambda$ is not an eigenvalue if and only if $z_{1}^{(n)}(s)$ diverges along the eigenspace $\operatorname{span}\left\{z_{1+}^{(n)}\right\}$ of $\Gamma_{+}^{(n)} s \rightarrow+\infty$.

Proof. Let $z_{1}(s), \ldots, z_{2 n}(s)$ be the solutions of (4.2) for which $\lim _{s \rightarrow+\infty}\left|z_{i}(s)\right| e^{-\Re \lambda_{i}^{+} s} s^{-l_{i}}$ exists and is non-zero for some non-negative integer $l_{i}$. Then $z_{1}^{(n)}$ is expanded as $z_{1}^{(n)}(s)=\sum_{\left(i_{1}, \ldots, i_{n}\right)} C_{i_{1}, \ldots, i_{n}} z_{i_{1}}(s) \wedge \cdots \wedge z_{i_{n}}(s)$ and $z_{2}^{(n)}$ is expressed as $z_{2}^{(n)}(s)=C z_{1}(s) \wedge \cdots \wedge z_{n}(s)$ for some non-zero constants $C_{i_{1}, \ldots, i_{n}}$ and $C$. By this expansion, $z_{1}^{(n)}(s) \wedge z_{2}^{(n)}(s) \neq 0$ is equvalent to $C_{n+1, \ldots, 2 n} \neq 0$ and this means that $z_{1}^{(n)}(s)$ diverges along the eigenspace $\operatorname{span}\left\{z_{1+}^{(n)}\right\}$ since $\lambda_{I}^{+}$is the eigenvalue of $\Gamma_{+}^{(n)}$ with largest real part.

In this paper, this problem is treated in the framework of the Graßmann manifold. Let $G_{n}\left(\mathbb{C}^{2 n}\right)$ be the Graßmann manifold of $n$-dimensional subspaces in $\mathbb{C}^{2 n}$. Then this manifold can be seen as a submanifold of the projective space $P\left(\stackrel{n}{\wedge} \mathbb{C}^{2 n}\right)$ of $\stackrel{n}{\wedge} \mathbb{C}^{2 n}$ by the following Plüker embedding:

$$
G_{n}\left(\mathbb{C}^{2 n}\right) \ni \operatorname{span}\left\{v_{1}, \ldots, v_{n}\right\} \mapsto\left[v_{1} \wedge \cdots \wedge v_{n}\right] \in P\left(\stackrel{n}{\wedge} \mathbb{C}^{2 n}\right)
$$

As the system (4.3) is linear, it induces a system on $G_{n}\left(\mathbb{C}^{2 n}\right)$ :

$$
\dot{\hat{z}}=\hat{\Gamma}(\hat{z} ; u(s), \Lambda ; c, \mu) \quad \hat{z} \in G_{n}\left(\mathbb{C}^{2 n}\right) \subset P\left(\stackrel{n}{\wedge} \mathbb{C}^{2 n}\right)
$$

Then lemma 4.3 is expressed as follows:

LEMMA 4.4. $\Lambda$ is not an eigenvalue if and only if

$$
\left[z_{1}^{(n)}(s ; \Lambda)\right] \rightarrow\left[z_{1+}^{(n)}\right] \quad(s \rightarrow+\infty)
$$

in $G_{n}\left(\mathbb{C}^{2 n}\right) \subset P\left(\stackrel{n}{\wedge} \mathbb{C}^{2 n}\right)$.

In what follows, the eigenvalue problem along the standing pulse solution, existence of which is proven in theorem 2, is treated from this point of view.

4.2. Analysis of Poincaré maps. The analysis makes use of the analysis of Poincaré maps. For this purpose, first coordinates and sections are suitably chosen. Let $(x, y)=\left(x_{1}, \bar{x}, y_{1}, \bar{y}\right)$ be a local coordinate system around $P$ in which system (2.6) satisfies Hypothesis 1 and is expressed as (2.2). Let $\Sigma_{i n}=\left\{x_{1}=\delta\right\}$ and $\Sigma_{\text {out }}=$ $\left\{y_{1}=\delta\right\}$ be local sections which are transversal to the heteroclinic orbits between $P$ and $Q$ which exist for $\left(c, \mu_{1}\right)=(0,0)$. Similarly, let $\left(x^{\prime}, y^{\prime}\right)=\left(x_{1}^{\prime}, \bar{x}^{\prime}, y_{1}^{\prime}, \bar{y}^{\prime}\right)$ be a local coordinate system around $Q$, and let $\Sigma_{\text {in }}^{\prime}=\left\{x_{1}^{\prime}=\delta\right\}$ and $\Sigma_{\text {out }}^{\prime}=\left\{y_{1}^{\prime}=\delta\right\}$ be local sections. As above (the discussion following Hypothesis 6 ), $\delta$ is assumed to be 1 through a scalar change of coordinates.

Consider the coupled system of (2.6) and (4.2)

$$
\left\{\begin{aligned}
\dot{w} & =F(w ; c, \mu) \\
\dot{z} & =\Gamma(w ; \Lambda ; c, \mu) z
\end{aligned}\right.
$$

where $w=(u, v)$. Let $(x, y, z)=\left(x_{1}, \bar{x}, y_{1}, \bar{y}, z_{(1)}, \ldots, z_{(2 n)}\right)$ and $\left(x^{\prime}, y^{\prime}, z^{\prime}\right)=$ $\left(x_{1}^{\prime}, \bar{x}^{\prime}, y_{1}^{\prime}, \bar{y}^{\prime}, z_{(1)}^{\prime}, \ldots, z_{(2 n)}^{\prime}\right)$ be local coordinate around $\{P\} \times \mathbb{C}^{2 n}$ and $\{Q\} \times \mathbb{C}^{2 n}$. By simple application of Kato [9] p. 99, these coordinates can be chosen such that 
they are analytic in $\Lambda$ and smooth in $c$ and $\mu$, and $\Gamma(w ; \Lambda, c, \mu)$ is expressed in the following form:

$$
\Gamma(w ; \Lambda ; c, \mu)=\left(\begin{array}{cccc}
-\lambda(\Lambda ; c, \mu) & 0 & 0 & 0 \\
0 & -A(\Lambda ; c, \mu) & 0 & 0 \\
0 & 0 & \lambda(\Lambda ;-c, \mu) & 0 \\
0 & 0 & 0 & A(\Lambda ;-c, \mu)
\end{array}\right)
$$

if $w$ is near $P$ and

$$
\Gamma(w ; \Lambda ; c, \mu)=\left(\begin{array}{cccc}
-\lambda^{\prime}(\Lambda ; c, \mu) & 0 & 0 & 0 \\
0 & -A^{\prime}(\Lambda ; c, \mu) & 0 & 0 \\
0 & 0 & \lambda^{\prime}(\Lambda ;-c, \mu) & 0 \\
0 & 0 & 0 & A^{\prime}(\Lambda ;-c, \mu)
\end{array}\right)
$$

if $w$ is near $Q$. Moreover $\Gamma$ and $D F$ coincide for $\Lambda=0$ i.e.

$$
\Gamma(w ; 0 ; c, \mu)=D F(w ; c, \mu)
$$

if $w$ is near $P$ or $Q$.

The system (4.5) induces a system on $\mathbb{R}^{2 n} \times \wedge \mathbb{C}^{2 n}$ in the same manner as (4.2) induces (4.3):

$$
\left\{\begin{aligned}
\dot{w} & =F(w ; c, \mu) \\
\dot{z}^{(n)} & =\Gamma^{(n)}(w ; \Lambda ; c, \mu) z^{(n)}
\end{aligned}\right.
$$

and this induces a system on $\mathbb{R}^{2 n} \times G_{n}\left(\mathbb{C}^{2 n}\right)$ :

$$
\left\{\begin{aligned}
\dot{w} & =F(w ; c, \mu) \\
\dot{\hat{z}} & =\hat{\Gamma}(w, \hat{z} ; \Lambda ; c, \mu)
\end{aligned}\right.
$$

Now consider the Poincaré maps:

$$
\begin{aligned}
& \Pi_{-}: \Sigma_{\text {out }}^{\prime} \times G_{n}\left(\mathbb{C}^{2 n}\right) \rightarrow \Sigma_{\text {in }} \times G_{n}\left(\mathbb{C}^{2 n}\right) \\
& \Pi_{0}: \Sigma_{\text {in }} \times G_{n}\left(\mathbb{C}^{2 n}\right) \rightarrow \Sigma_{\text {out }} \times G_{n}\left(\mathbb{C}^{2 n}\right) \\
& \Pi_{+}: \Sigma_{\text {out }} \times G_{n}\left(\mathbb{C}^{2 n}\right) \rightarrow \Sigma_{\text {in }}^{\prime} \times G_{n}\left(\mathbb{C}^{2 n}\right)
\end{aligned}
$$

$\Pi_{*}(*= \pm, 0)$ is denoted as:

$$
\Pi_{*}(w, \hat{z} ; \Lambda ; c, \mu)=\left(\pi_{*}(w, c, \mu), \hat{\pi}_{*}(w, \hat{z} ; \Lambda ; c, \mu)\right)
$$

On each section, inhomogeneous coordinate systems on $P\left(\wedge \wedge^{2 n}\right)$ are employed as coordinate systems on $G_{n}\left(\mathbb{C}^{2 n}\right)$. On $\Sigma_{\text {out }}^{\prime} \times G_{n}\left(\mathbb{C}^{2 n}\right)$, let

$$
Z^{\prime}=\left(Z_{1, n+2, \ldots, 2 n}^{\prime}, \ldots, Z_{i_{1}, \ldots, i_{n}}^{\prime}, \ldots\right)
$$

be the inhomogeneous coordinate systems on $P\left(\wedge^{n} \mathbb{C}^{2 n}\right)$ centered at $z_{(n+1)}^{\prime} \wedge \cdots \wedge z_{(2 n)}^{\prime}$, that is, the point

$$
\left[\sum_{\left(i_{1}, \ldots, i_{n}\right)} C_{i_{1}, \ldots, i_{n}}^{\prime} z_{\left(i_{1}\right)}^{\prime} \wedge \cdots \wedge z_{\left(i_{n}\right)}^{\prime}\right]
$$


is expressed as

$$
Z^{\prime}=\left(\frac{C_{1, n+2, \ldots, 2 n}^{\prime}}{C_{n+1, \ldots, 2 n}^{\prime}}, \ldots, \frac{C_{i_{1}, \ldots, i_{n}}^{\prime}}{C_{n+1, \ldots, 2 n}^{\prime}}, \ldots\right)
$$

Then $\left(x_{1}^{\prime}, \bar{x}^{\prime}, \bar{y}^{\prime}, Z^{\prime}\right)$ is employed as the coordinate system on $\Sigma_{\text {out }}^{\prime} \times G_{n}\left(\mathbb{C}^{2 n}\right)$. Similarly, $\left(x_{1}, \bar{x}, \bar{y}, Z\right)$ is employed as the coordinate system on $\Sigma_{\text {out }} \times G_{n}\left(\mathbb{C}^{2 n}\right)$.

On $\Sigma_{i n} \times G_{n}\left(\mathbb{C}^{2 n}\right)$, let $\zeta=\left(\zeta_{n+1, \ldots, 2 n}, \ldots, \zeta_{i_{1}, \ldots, i_{n}}, \ldots\right)$ be the inhomogeneous coordinate system on $P\left(\wedge \mathbb{C}^{2 n}\right)$ centered at $z_{(1)} \wedge z_{(n+2)} \wedge \cdots \wedge z_{(2 n)}$, that is, the point

$$
\left[\sum_{\left(i_{1}, \ldots, i_{n}\right)} C_{i_{1}, \ldots, i_{n}} z_{\left(i_{1}\right)} \wedge \cdots \wedge z_{\left(i_{n}\right)}\right]
$$

is expressed as

$$
\zeta=\left(\frac{C_{n+1, \ldots, 2 n}}{C_{1, n+2, \ldots, 2 n}}, \ldots, \frac{C_{i_{1}, \ldots, i_{n}}}{C_{1, n+2, \ldots, 2 n}}, \ldots\right)
$$

Then $\left(\bar{x}, y_{1}, \bar{y}, \zeta\right)$ is employed as the coordinate system on $\Sigma_{i n} \times G_{n}\left(\mathbb{C}^{2 n}\right)$.

In what follows, the map $\hat{\pi}_{*}(*= \pm, 0)$ is analysed.

As the system (4.6) is linear around $\{P\} \times \wedge \mathbb{C}^{2 n}, \hat{\pi}_{0}$ is obtained by direct calculation.

LEMMA 4.5 .

$$
\hat{\pi}_{0}\left(\bar{x}, y_{1}, \bar{y}, \zeta_{n+1, \ldots, 2 n}, \ldots\right)=\left(\zeta_{n+1, \ldots, 2 n}^{-1} y_{1}^{\frac{\lambda(\Lambda ; c, \mu)+\lambda(\Lambda ;-c, \mu)}{\lambda(0 ; c, \mu)}}, \ldots, Z_{i_{1}, \ldots, i_{n}}, \ldots\right)
$$

and for $\left(i_{1}, \ldots, i_{n}\right) \neq(1, n+2, \ldots, 2 n)$ the following estimate holds:

$$
\left|Z_{i_{1}, \ldots, i_{n}}\right|<\zeta_{n+1, \ldots, 2 n}^{-1} \zeta_{i_{1}, \ldots, i_{n}} y_{1}^{\frac{\lambda(\Lambda ; c, \mu)+\nu}{\lambda(0 ; c, \mu)}}
$$

where $\nu$ is what appears in Hypothesis 4.

As for $\hat{\pi}_{-}$, consider its Taylor expansion:

$$
\begin{gathered}
\hat{\pi}_{-}\left(x^{\prime}, y^{\prime} ; Z^{\prime} ; \Lambda ; 0, \mu\right)=\hat{\pi}_{-}\left(0, \mathrm{y}^{\prime}\left(0, \mu_{2}\right) ; 0 ; 0 ; 0,0, \mu_{2}\right) \\
+\frac{\partial \hat{\pi}_{-}}{\partial Z^{\prime}} Z^{\prime}+\frac{\partial \hat{\pi}_{-}}{\partial \Lambda} \Lambda+\frac{\partial^{2} \hat{\pi}_{-}}{\partial \Lambda^{2}} \Lambda^{2} \\
+\frac{\partial \hat{\pi}_{-}}{\partial\left(x^{\prime}, y^{\prime}\right)}\left(x^{\prime}, y^{\prime}-\mathrm{y}^{\prime}\left(0, \mu_{2}\right)\right)+\frac{\partial \hat{\pi}_{-}}{\partial \mu_{1}} \mu_{1} \\
+(\text { higher order terms })
\end{gathered}
$$

where $\left(0, \mathrm{y}^{\prime}(\mu)\right):=\pi_{-}^{-1}\left(1, \xi\left(\bar{y}(\mu) ; 0, \mu_{1}, \mu_{2}\right), \eta_{1}\left(\bar{y}(\mu) ; 0, \mu_{1}, \mu_{2}\right), \bar{y}(\mu)\right)$ for the function $\bar{y}(\mu)$ defined in proposition 3.2

LEMMA 4.6 .

$$
\left(\hat{\pi}_{-}\right)_{n+1, \ldots, 2 n}\left(0, \mathrm{y}^{\prime}\left(0, \mu_{2}\right) ; 0 ; 0 ; 0,0, \mu_{2}\right)=0
$$

and

$$
\frac{\partial\left(\hat{\pi}_{-}\right)_{n+1, \ldots, 2 n}}{\partial Z_{1, n+2, \ldots, 2 n}^{\prime}}\left(0, \mathrm{y}^{\prime}\left(0, \mu_{2}\right) ; 0 ; 0 ; 0,0, \mu_{2}\right)=-1
$$


where $\left(\hat{\pi}_{-}\right)_{n+1, \ldots, 2 n}$ stands for the $(n+1, \ldots, 2 n)$ component of $\hat{\pi}_{-}$.

Proof. The first equality is clear from the fact that $\left(0, \mathrm{y}^{\prime}\left(0, \mu_{2}\right)\right)$ is on the heteroclinic orbit from $Q$ to $P$ and $\Lambda=0$ is an eigenvalue, and lemma 4.3.

The second equality is proven as follows.

As the system is symmetric when $\mu_{1}=0$ (Hypothesis 10), there exists a solution $\left(w_{0}(s), z_{0}(s)\right)$ of $(4.5)$ for $\Lambda=c=\mu_{1}=0$ such that $w_{0}(s)$ is a heteroclinic solution from $Q$ to $P$, and if $\left(u_{0}(s), v_{0}(s)\right)$ are the $(u, v)$ coordinates of $w_{0}(s)$ and $\left(p_{0}(s), q_{0}(s)\right)$ are $z_{0}(s)$ in $(p, q)$ coordinates then $\left(u_{0}(-s), v_{0}(-s), p_{0}(-s), q_{0}(-s)\right)=$ $\left(-u_{0}(s), v_{0}(s),-p_{0}(s), q_{0}(s)\right)$ holds and $\lim _{s \rightarrow \pm \infty}\left|z_{0}(s)\right| e^{\mp \lambda\left(0 ; 0, \mu_{2}\right) s}$ exist and are nonzero. See [13]. Also Hypothesis 6 implies that $\lim _{s \rightarrow \pm \infty}\left|\dot{w}_{0}(s)\right| e^{ \pm \lambda\left(0 ; 0, \mu_{2}\right) s}$ exist and are non-zero.

Chose $s_{0}$ such that $w_{0}\left(-s_{0}\right) \in \Sigma_{\text {out }}^{\prime}$ and $w\left(s_{0}\right) \in \Sigma_{\text {in }}$ and let

$$
z_{0}\left(-s_{0}\right)=\left(z_{(1)}^{\prime}\left(-s_{0}\right), \ldots, z_{(n)}^{\prime}\left(-s_{0}\right), z_{(n+1)}^{\prime}\left(-s_{0}\right), \ldots, z_{(2 n)}^{\prime}\left(-s_{0}\right)\right)
$$

then

$$
\begin{aligned}
z_{0}\left(s_{0}\right) & =\left(z_{(1)}\left(s_{0}\right), \ldots, z_{(n)}\left(s_{0}\right), z_{(n+1)}\left(s_{0}\right), \ldots, z_{(2 n)}\left(s_{0}\right)\right) \\
& =\left(z_{(n+1)}^{\prime}\left(-s_{0}\right), \ldots, z_{(2 n)}^{\prime}\left(-s_{0}\right), z_{(1)}^{\prime}\left(-s_{0}\right), \ldots, z_{(n)}^{\prime}\left(-s_{0}\right)\right)
\end{aligned}
$$

by the symmetry (Hypothesis 1 and 10$)$, and $z_{(1)}^{\prime}\left(-s_{0}\right) \neq 0, z_{(2)}^{\prime}\left(-s_{0}\right)=\cdots=$ $z_{(n)}^{\prime}\left(-s_{0}\right)=0$ holds. Also, let

$$
\dot{w}_{0}\left(-s_{0}\right)=\left(\dot{w}_{(1)}^{\prime}\left(-s_{0}\right), \ldots, \dot{w}_{(n)}^{\prime}\left(-s_{0}\right), \dot{w}_{(n+1)}^{\prime}\left(-s_{0}\right), \ldots, \dot{w}_{(2 n)}^{\prime}\left(-s_{0}\right)\right)
$$

in $\left(z_{(1)}^{\prime}, \ldots, z_{(2 n)}^{\prime}\right)$ coordinates then;

$$
\begin{aligned}
\dot{w}_{0}\left(s_{0}\right) & =\left(\dot{w}_{(1)}\left(s_{0}\right), \ldots, \dot{w}_{(n)}\left(s_{0}\right), \dot{w}_{(n+1)}\left(s_{0}\right), \ldots, \dot{w}_{(2 n)}\left(s_{0}\right)\right) \\
& =\left(-\dot{w}_{(n+1)}^{\prime}\left(-s_{0}\right), \ldots,-\dot{w}_{(2 n)}^{\prime}\left(-s_{0}\right),-\dot{w}_{(1)}^{\prime}\left(-s_{0}\right), \ldots,-\dot{w}_{(n)}^{\prime}\left(-s_{0}\right)\right)
\end{aligned}
$$

in $\left(z_{(1)}, \ldots, z_{(2 n)}\right)$ coordinates, and $\dot{w}_{(n+1)}^{\prime}\left(-s_{0}\right) \neq 0, \dot{w}_{(1)}^{\prime}\left(-s_{0}\right)=\cdots=\dot{w}_{(n)}^{\prime}\left(-s_{0}\right)=$ 0 .

Consider a system on $\mathbb{R}^{2 n} \times \stackrel{n-1}{\wedge} \mathbb{C}^{2 n}$ :

$$
\begin{cases}\dot{w} & =F(w ; c, \mu) \\ \dot{z}^{(n-1)} & =\Gamma^{(n-1)}(w ; \Lambda ; c, \mu) z^{(n-1)}\end{cases}
$$

Hypothesis 7 implies that there exists a solution $\left(w_{0}(s), z_{0}^{(n-1)}(s)\right)$ of $(4.8)$ such that $\lim _{s \rightarrow-\infty}\left|z_{0}^{(n-1)}(s)\right| e^{-\lambda^{(n-1) '} s}$ and $\lim _{s \rightarrow+\infty}\left|z_{0}^{(n-1)}(s)\right| e^{-\lambda^{(n-1)} s}$ exist and are non-zero, where $\lambda^{(n-1)^{\prime}}$ and $\lambda^{(n-1)}$ are the sum of all eigenvalues of $A^{\prime}\left(0 ; 0,0, \mu_{2}\right)$ and $A\left(0 ; 0,0, \mu_{2}\right)$. Then $z_{0}^{(n-1)}\left(-s_{0}\right)=C^{\prime} z_{(n+2)}^{\prime} \wedge \cdots \wedge z_{(2 n)}^{\prime}$ and $z_{0}^{(n-1)}\left(s_{0}\right)=\sum_{\left(i_{1}, \ldots i_{n-1}\right)} C_{i_{1}, \ldots, i_{n-1}} z_{\left(i_{1}\right)} \wedge$ $\cdots \wedge z_{\left(i_{n-1}\right)}$ with $C_{n+2, \ldots, 2 n} \neq 0$.

Let $z_{0}^{(n)}(s ; \zeta):=\dot{w}_{0}(s) \wedge z_{0}^{(n-1)}(s)+\zeta z_{0}(s) \wedge z_{0}^{(n-1)}(s)$; then $\left(w_{0}(s), z_{0}^{(n)}(s)\right)$ satisfies (4.6) and

$$
\begin{aligned}
z_{0}^{(n)}\left(-s_{0} ; \zeta\right) & =\left(\sum C_{i}^{\prime} z_{(i)}^{\prime}\right) \wedge C^{\prime} z_{(n+2)} \wedge \cdots \wedge z_{(2 n)}+\zeta\left(\sum D_{i}^{\prime} z_{(i)}^{\prime}\right) \wedge C^{\prime} z_{(n+2)} \wedge \cdots \wedge z_{(2 n)} \\
& =\left(C_{n+1}^{\prime}+\zeta D_{n+1}^{\prime}\right) C^{\prime} z_{(n+1)} \wedge \cdots \wedge z_{(2 n)}+\zeta D_{1}^{\prime} C^{\prime} z_{(1)} \wedge z_{(n+2)} \wedge \cdots \wedge z_{(2 n)}
\end{aligned}
$$


since $C_{1}^{\prime}=\cdots=C_{n}^{\prime}=0, C_{n+1}^{\prime} \neq 0$ and $D_{1}^{\prime} \neq 0, D_{2}^{\prime}=\cdots=D_{n}^{\prime}=0$, and

$$
\begin{aligned}
& z_{0}^{(n)}\left(s_{0} ; \zeta\right) \\
& =\left(\sum_{i=1}^{n}-C_{i+n}^{\prime} z_{(i)}+\sum_{i=n+1}^{2 n}-C_{i-n}^{\prime} z_{(i)}\right) \wedge \sum_{\left(i_{1}, \ldots i_{n-1}\right)} C_{i_{1}, \ldots, i_{n-1}} z_{\left(i_{1}\right)} \wedge \cdots \wedge z_{\left(i_{n-1}\right)} \\
& \quad+\zeta\left(\sum_{i=1}^{n} D_{i+n}^{\prime} z_{(i)}+\sum_{i=n+1}^{2 n} D_{i-n}^{\prime} z_{(i)}\right) \wedge \sum_{\left(i_{1}, \ldots i_{n-1}\right)} C_{i_{1}, \ldots, i_{n-1}} z_{\left(i_{1}\right)} \wedge \cdots \wedge z_{\left(i_{n-1}\right)} \\
& =\zeta D_{1}^{\prime} C_{n+2, \ldots, 2 n} z_{(n+1)} \wedge \cdots \wedge z_{(2 n)} \\
& \quad+\left(-C_{n+1}^{\prime}+\zeta D_{n+1}^{\prime}\right) C_{n+2, \ldots, 2 n} z_{(1)} \wedge z_{(n+2)} \wedge \cdots \wedge z_{(2 n)} \\
& \quad+\cdots
\end{aligned}
$$

Thus

$$
\left(\hat{\pi}_{-}\right)_{n+1, \ldots, 2 n}\left(\frac{\zeta D_{1}^{\prime}}{C_{n+1}^{\prime}+\zeta D_{n+1}^{\prime}}, 0, \ldots, 0\right)=\frac{-\zeta D_{1}^{\prime}}{C_{n+1}^{\prime}-\zeta D_{n+1}^{\prime}}
$$

and the second equality is obtained from this.

LEMMA 4.7.

$$
\frac{\partial\left(\hat{\pi}_{-}\right)_{n+1, \ldots, 2 n}}{\partial \mu_{1}}\left(0, \mathrm{y}^{\prime}\left(0, \mu_{2}\right) ; 0 ; 0 ; 0,0, \mu_{2}\right)=-1
$$

Proof. Let $w_{0}\left(s ; \mu_{1}\right)$ be a solution of $(2.6)$ with the initial condition

$$
w_{0}\left(s_{0}\left(\mu_{1}\right), \mu_{1}\right)=\left(1, \xi\left(0 ; 0, \mu_{1}, \mu_{2}\right), \eta_{1}\left(0 ; 0, \mu_{1}, \mu_{2}\right), 0\right)
$$

where $\xi$ and $\eta_{1}$ are as in hypothesis $7, s_{0}\left(\mu_{1}\right)$ is chosen such that $w_{0}\left(s_{0}\left(\mu_{1}\right), \mu_{1}\right) \in$ $\Sigma_{\text {in }}$ and $w_{0}\left(-s_{0}\left(\mu_{1}\right), \mu_{1}\right) \in \Sigma_{\text {out }}^{\prime}$. Then $\dot{w}_{0}\left(s_{0}\left(\mu_{1}\right), \mu_{1}\right)=\left(-\lambda,-A \xi, \lambda \eta_{1}, 0\right)$. Here, $\eta_{1}\left(0 ; 0, \mu_{1}, \mu_{2}\right)=\mu_{1}+O\left(\mu_{1}^{2}\right)$. Let $z^{2}\left(s ; \mu_{1}\right), \ldots, z^{n}\left(s ; \mu_{1}\right) \in T_{w_{0}\left(s_{0}\left(\mu_{1}\right), \mu_{1}\right)} W^{u}(Q)$ be linearly independent solutions of (4.5) with $\Lambda=0$ satisfying $z_{(n+j)}^{i}\left(s_{0}\left(\mu_{1}\right), \mu_{1}\right)=\delta_{i, j}$ $(j=2, \ldots, n)$ and $z_{(1)}^{i}\left(s_{0}\left(\mu_{1}\right), \mu_{1}\right)=0$, where $z_{(k)}^{i}\left(s_{0}\left(\mu_{1}\right), \mu_{1}\right)$ is the $z_{(k)}$ component of $z^{i}\left(s_{0}\left(\mu_{1}\right), \mu_{1}\right)$. Also let $\check{z}^{(n)}\left(s ; \mu_{1}\right)=\dot{w}_{0}\left(s ; \mu_{1}\right) \wedge z^{2}\left(s ; \mu_{1}\right) \wedge \cdots \wedge z^{n}\left(s ; \mu_{1}\right)$. Then $\left(w_{0}\left(s, \mu_{1}\right), \check{z}^{(n)}\left(s ; \mu_{1}\right)\right)$ is a solution of $(4.6)$, and

$$
\begin{aligned}
\check{z}^{(n)}\left(-s_{0}\left(\mu_{1}\right), \mu_{1}\right)= & C^{\prime} z_{(n+1)}^{\prime} \wedge \cdots \wedge z_{(2 n)}^{\prime} \\
\check{z}^{(n)}\left(s_{0}\left(\mu_{1}\right), \mu_{1}\right)=- & \lambda z_{(1)} \wedge z_{(n+2)} \wedge \cdots \wedge z_{(2 n)} \\
& \quad+\left(\lambda \mu_{1}+O\left(\mu_{1}^{2}\right)\right) z_{(n+1)} \wedge \cdots \wedge z_{(2 n)}+\cdots
\end{aligned}
$$

The conclusion follows from this.

LEMMA 4.8.

$$
\frac{\partial\left(\hat{\pi}_{-}\right)_{n+1, \ldots, 2 n}}{\partial \Lambda}\left(0, \mathrm{y}^{\prime}\left(0, \mu_{2}\right) ; 0 ; 0 ; 0,0, \mu_{2}\right)=-\frac{1}{\lambda\left(0 ; 0,0, \mu_{2}\right)} \mu_{2}
$$

and

$$
\frac{\partial^{2}\left(\hat{\pi}_{-}\right)_{n+1, \ldots, 2 n}}{\partial \Lambda^{2}}\left(0, \mathrm{y}^{\prime}\left(0, \mu_{2}\right) ; 0 ; 0 ; 0,0, \mu_{2}\right)<0
$$


Proof. Let $w_{1}\left(s ; c, \mu_{2}\right) \in W^{u}(Q)$ be a solution of $(2.6)$ with the initial condition $w_{1}\left(s ; c, \mu_{2}\right)=\left(1, \xi\left(0 ; c, 0, \mu_{2}\right), \eta_{1}\left(0 ; c, 0, \mu_{2}\right), 0\right)$. Also let $\left(w_{1}\left(s ; 0, \mu_{2}\right), \check{z}\left(s ; \Lambda, \mu_{2}\right)\right)$ be a solution of (4.5) such that $\lim _{s \rightarrow-\infty}\left|\check{z}\left(s ; \Lambda, \mu_{2}\right)\right| e^{-\lambda^{\prime} s}$ exists and is non-zero, $\check{z}\left(s ; \Lambda, \mu_{2}\right)$ is analytic in $\Lambda$ and smooth in $\mu_{2}$, and $\check{z}\left(s ; 0, \mu_{2}\right)=\dot{w}_{1}\left(s ; 0, \mu_{2}\right)$. Then

$$
\left(w_{1}\left(s ; 0, \mu_{2}\right), \frac{\partial \check{z}}{\partial \Lambda}\left(s ; 0, \mu_{2}\right)-\frac{\partial w_{1}}{\partial c}\left(s ; 0, \mu_{2}\right)\right)
$$

satisfies (4.5). This is easily seen by subtracting the differentiation of (2.6) with respect to $c$ from the differentiation of (4.5) with respect to $\Lambda$. Moreover,

$$
\frac{\partial \check{z}}{\partial \Lambda}\left(s ; 0, \mu_{2}\right)-\frac{\partial w_{1}}{\partial c}\left(s ; 0, \mu_{2}\right) \in T_{w_{1}\left(s ; 0, \mu_{2}\right)} W^{u}(Q)
$$

because

$$
\lim _{s \rightarrow-\infty}\left\{\frac{\partial \check{z}}{\partial \Lambda}\left(s ; 0, \mu_{2}\right)-\frac{\partial w_{1}}{\partial c}\left(s ; 0, \mu_{2}\right)\right\}=0
$$

Let $\left(w_{1}\left(s ; 0, \mu_{2}\right), \check{z}^{(n-1)}\left(s ; \Lambda, \mu_{2}\right)\right)$ be a solution of $(4.8)$ for which

$$
\lim _{s \rightarrow-\infty}\left|\check{z}^{(n-1)}\left(s ; \Lambda, \mu_{2}\right)\right| e^{-\lambda^{(n-1)^{\prime}} s}
$$

exists, is non-zero, and is analytic in $\Lambda$. Then $\left(w_{1}\left(s ; 0, \mu_{2}\right), \check{z}^{(n)}\left(s ; \Lambda, \mu_{2}\right)\right)$ is a solution of (4.6) for $\check{z}^{(n)}\left(s ; \Lambda, \mu_{2}\right):=\check{z}\left(s ; \Lambda, \mu_{2}\right) \wedge \check{z}^{(n-1)}\left(s ; \Lambda, \mu_{2}\right)$, and

$$
\lim _{s \rightarrow-\infty}\left|\check{z}^{(n)}\left(s ; \Lambda, \mu_{2}\right)\right| e^{-\left\{\lambda^{(n-1)^{\prime}}+\lambda^{\prime}\right\} s}
$$

exists.

If $\check{z}\left(s_{0} ; \Lambda, \mu_{2}\right)$ and $\check{z}^{(n-1)}\left(s_{0} ; \Lambda, \mu_{2}\right)$ are expanded in $\left(z_{(1)}, \ldots, z_{(2 n)}\right)$ coordinates:

$$
\begin{aligned}
\check{z}\left(s_{0} ; \Lambda, \mu_{2}\right) & =\left(-\lambda(\Lambda), C_{2}(\Lambda), \ldots, C_{n+1}(\Lambda), \ldots, C_{2 n}(\Lambda)\right) \\
\check{z}^{(n-1)}\left(s_{0} ; \Lambda, \mu_{2}\right) & =\sum_{\left(i_{1}, \ldots, i_{n-1}\right)} C_{i_{1}, \ldots, i_{n-1}}(\Lambda) z_{\left(i_{1}\right)} \wedge \cdots \wedge z_{\left(i_{n-1}\right)}
\end{aligned}
$$

then

$$
\begin{aligned}
& \check{z}^{(n)}\left(s_{0} ; \Lambda, \mu_{2}\right) \\
& =\left(-\lambda(\Lambda) C_{n+2, \ldots, 2 n}(\Lambda)\right. \\
& \left.+\sum_{i=n+2}^{2 n} C_{i}(\Lambda) C_{1, n+2, \ldots, i-1, i+1, \ldots, 2 n}(\Lambda)\right) z_{(1)} \wedge z_{(n+2)} \wedge \cdots \wedge z_{(2 n)} \\
& \quad+\left(\sum_{i=n+1}^{2 n} C_{i}(\Lambda) C_{n+1, \ldots, i-1, i+1, \ldots, 2 n}(\Lambda)\right) z_{(n+1)} \wedge \cdots \wedge z_{(2 n)} \\
& \quad+\cdots
\end{aligned}
$$

Here, (4.11) and

$$
\check{z}^{(n-1)}\left(s_{0} ; 0, \mu_{2}\right) \in \stackrel{n-1}{\wedge} T_{w_{1}\left(s_{0} ; 0, \mu_{2}\right)} W^{u}(Q)
$$


mean

$$
\left(\left(\frac{\partial \check{z}}{\partial \Lambda}\left(s ; 0, \mu_{2}\right)-\frac{\partial w_{1}}{\partial c}\left(s ; 0, \mu_{2}\right)\right) \wedge \check{z}^{(n-1)}\left(s ; 0, \mu_{2}\right)\right)_{n+1, \ldots, 2 n}=0
$$

and

$$
\check{z}\left(s_{0} ; 0, \mu_{2}\right) \in T_{w_{1}\left(s_{0} ; 0, \mu_{2}\right)} W^{u}(Q) \cap T_{w_{1}\left(s_{0} ; 0, \mu_{2}\right)} W^{s}(P)
$$

means

$$
\left(\check{z}\left(s ; 0, \mu_{2}\right) \wedge \frac{\partial \check{z}^{(n-1)}}{\partial \Lambda}\left(s ; 0, \mu_{2}\right)\right)_{n+1, \ldots, 2 n}=0
$$

where ()$_{n+1, \ldots, 2 n}$ stands for $\left(z_{(n+1)} \wedge \cdots \wedge z_{(2 n)}\right)$ component. Thus

$$
\begin{aligned}
\frac{\partial \check{z}^{(n)}}{\partial \Lambda}\left(s_{0} ; 0, \mu_{2}\right) & =\frac{\partial \check{z}}{\partial \Lambda}\left(s_{0} ; 0, \mu_{2}\right) \wedge \check{z}^{(n-1)}\left(s_{0} ; 0, \mu_{2}\right)+\check{z}\left(s_{0} ; 0, \mu_{2}\right) \wedge \frac{\partial \check{z}^{(n-1)}}{\partial \Lambda}\left(s_{0} ; 0, \mu_{2}\right) \\
& =\frac{\partial \eta_{1}}{\partial c}\left(0,0, \mu_{2}\right) z_{(n+1)} \wedge \cdots \wedge z_{(2 n)}+\cdots
\end{aligned}
$$

Therefore

$$
\begin{aligned}
& \left.\frac{\partial\left(\pi_{-}\right)_{n+1, \ldots, 2 n}}{\partial \Lambda}\right|_{\Lambda=0} \\
& =\left.\frac{\partial}{\partial \Lambda}\left(\frac{\sum_{i=n+1}^{2 n} C_{i}(\Lambda) C_{n+1, \ldots, i-1, i+1, \ldots, 2 n}(\Lambda)}{-\lambda(\Lambda) C_{n+2, \ldots, 2 n}(\Lambda)+\sum_{i=n+2}^{2 n} C_{i}(\Lambda) C_{1, n+2, \ldots, i-1, i+1, \ldots, 2 n}(\Lambda)}\right)\right|_{\Lambda=0} \\
& =-\frac{1}{\lambda\left(0 ; 0,0, \mu_{2}\right)} \frac{\partial \eta_{1}}{\partial c}\left(0,0, \mu_{2}\right)
\end{aligned}
$$

since $C_{i}(0)=0$ for $i=n+1, \ldots, 2 n$. (4.9) follows from this and

$$
\eta_{1}\left(0 ; c, \mu_{1}, \mu_{2}\right)=\mu_{1}+c \mu_{2}+c^{3}+o\left(\left|\mu_{1}\right|+\left|c^{2} \mu_{2}\right|+\left|c^{4}\right|\right)
$$

As for $(4.10)$, let $\left(w_{1}\left(s ; 0, \mu_{2}\right), \check{z}_{2}^{(n)}\left(s ; \Lambda, \mu_{2}\right)\right)$ be a solution of $(4.6)$ for which

$$
\lim _{s \rightarrow+\infty}\left|\check{z}_{2}^{(n)}\left(s ; \Lambda, \mu_{2}\right)\right| e^{\left\{\lambda^{n-1}+\lambda\right\} s}
$$

exists and is non-zero, and is analytic in $\Lambda$, then

$$
\check{z}_{2}^{(n)}\left(s_{0} ; \Lambda, \mu_{2}\right)=\check{C}(\Lambda) z_{(1)} \wedge \cdots \wedge z_{(n)}
$$

Define an Evans' function $D(\Lambda)$ by:

$$
D(\Lambda):=\check{z}^{(n)}\left(s_{0} ; \Lambda, \mu_{2}\right) \wedge \check{z}_{2}^{(n)}\left(s_{0} ; \Lambda, \mu_{2}\right)
$$

Then $D(\Lambda)=0$ if and only if $\Lambda$ is an eigenvalue. (See [1].)

By (4.12),

$$
D(\Lambda)=\check{C}(\Lambda)\left(\sum_{i=n+1}^{2 n} C_{i}(\Lambda) C_{n+1, \ldots, i-1, i+1, \ldots, 2 n}(\Lambda)\right)
$$


and

$$
\begin{aligned}
\left.\frac{d D}{d \Lambda}\right|_{\Lambda=0} & =\left.\check{C}(0) \frac{d}{d \Lambda}\right|_{\Lambda=0}\left(\sum_{i=n+1}^{2 n} C_{i}(\Lambda) C_{n+1, \ldots, i-1, i+1, \ldots, 2 n}(\Lambda)\right) \\
& =\check{C}(0) \frac{\partial \eta_{1}}{\partial c}\left(0,0, \mu_{2}\right)
\end{aligned}
$$

Thus $\left.\frac{d D}{d \Lambda}\right|_{\Lambda=0}=0$ if and only if $\frac{\partial \eta_{1}}{\partial c}\left(0,0, \mu_{2}\right)=0$.

If $\left.\frac{d D}{d \Lambda}\right|_{\Lambda=0}=0$, then

$$
\left.\frac{d^{2} D}{d \Lambda^{2}}\right|_{\Lambda=0}=\left.\check{C}(0) \frac{d^{2}}{d \Lambda^{2}}\right|_{\Lambda=0}\left(\sum_{i=n+1}^{2 n} C_{i}(\Lambda) C_{n+1, \ldots, i-1, i+1, \ldots, 2 n}(\Lambda)\right)
$$

On the other hand, hypothesis 11 implies that there are two zeros of $D(\Lambda)$ for $\Re \Lambda>\sigma_{1}$ counting multiplicity. Moreover, if $\check{z}^{(n)}$ is chosen such that $\lim _{\Lambda \rightarrow+\infty} D(\Lambda)>0$, then $\left.\frac{d D}{d \Lambda}\right|_{\Lambda=0}>0$ if and only if $\frac{\partial \eta_{1}}{\partial c}>0,\left.\frac{d D}{d \Lambda}\right|_{\Lambda=0}<0$ if and only if $\frac{\partial \eta_{1}}{\partial c}<0$, and $\left.\frac{d^{2} D}{d \Lambda^{2}}\right|_{\Lambda=0}>0$ when $\frac{\partial \eta_{1}}{\partial c}=0$. Thus, this choice of $\check{z}_{2}^{(n)}$ corresponds to $\check{C}(0)>0$ and

$$
\left.\frac{d^{2}}{d \Lambda^{2}}\right|_{\Lambda=0}\left(\sum_{i=n+1}^{2 n} C_{i}(\Lambda) C_{n+1, \ldots, i-1, i+1, \ldots, 2 n}(\Lambda)\right)>0
$$

Therefore at $\mu_{2}=0$,

$$
\begin{aligned}
& \frac{\partial^{2}\left(\hat{\pi}_{-}\right)_{n+1, \ldots, 2 n}}{\partial \Lambda^{2}}\left(0, \mathrm{y}^{\prime}\left(0, \mu_{2}\right) ; \mathrm{Z}^{\prime}\left(\mu_{2}\right) ; 0 ; 0,0,0\right) \\
& =-\left.\frac{1}{\lambda\left(0 ; 0,0, \mu_{2}\right)} \frac{d^{2}}{d \Lambda^{2}}\right|_{\Lambda=0}\left(\sum_{i=n+1}^{2 n} C_{i}(\Lambda) C_{n+1, \ldots, i-1, i+1, \ldots, 2 n}(\Lambda)\right) \\
& <0
\end{aligned}
$$

LEMMA 4.9.

$$
\hat{\pi}_{+}\left(u, v, \hat{z} ; c, \mu_{1}, \mu_{2}\right)=\hat{\pi}_{-}\left(-u,-v, \hat{z} ; c,-\mu_{1}, \mu_{2}\right)
$$

Proof. Under Hypothesis 10, the system 4.6 is invariant under the transformation $\left(u, v, \mu_{1}\right) \rightarrow\left(-u,-v,-\mu_{1}\right)$ i.e.

$$
\begin{cases}F\left(w ; c, \mu_{1}, \mu_{2}\right) & =-F\left(-w ; c,-\mu_{1}, \mu_{2}\right) \\ \Gamma\left(w ; \Lambda ; c, \mu_{1}, \mu_{2}\right) & =\Gamma\left(-w ; \Lambda ; c,-\mu_{1}, \mu_{2}\right)\end{cases}
$$

Thus if $w_{0}\left(s ; c, \mu_{1}, \mu_{2}\right)$ is the heteroclinic solution of

$$
\dot{w}=F\left(w ; c, \mu_{1}, \mu_{2}\right)
$$

from $Q$ to $P$, then $-w\left(s ; c, \mu_{1}, \mu_{2}\right)$ is the heteroclinic solution of

$$
\dot{w}=F\left(w ; c,-\mu_{1}, \mu_{2}\right)
$$

from $P$ to $Q$, and the Poincaré map has the following relation:

$$
\begin{aligned}
& \left(\pi_{+}\left(u, v ; c, \mu_{1}, \mu_{2}\right), \hat{\pi}_{+}\left(u, v, \hat{z} ; c, \mu_{1}, \mu_{2}\right)\right) \\
& \quad=\left(\pi_{-}\left(-u,-v ; c,-\mu_{1}, \mu_{2}\right), \hat{\pi}_{-}\left(-u,-v, \hat{z} ; c,-\mu_{1}, \mu_{2}\right)\right)
\end{aligned}
$$

This includes the lemma. $\square$ 
4.3. Proof of theorem 3. Theorem 3 is proven by tracing the image of the unstable manifold of $Q$ under the Poincaré maps.

Let $w(s ; \mu)$ be the homoclinic solution of $(2.6)$ to $Q$ with $w\left(s_{1} ; \mu\right) \in \Sigma_{\text {out }}^{\prime}, w\left(s_{2} ; \mu\right)$ $\in \Sigma_{\text {in }}$ and $w\left(s_{3} ; \mu\right) \in \Sigma_{\text {out }}$. Consider the image of $\left(w\left(s_{1} ; \mu\right),\left[z_{(n+1)} \wedge \cdots \wedge z_{(2 n)}\right]\right)$ under the Poincaré maps $\Pi_{-}, \Pi_{0}$ and $\Pi_{+}$.

First, by lemmas $4.6,4.7,4.8$ and the fact that $\frac{\partial \mathrm{y}^{\prime}}{\partial \mu_{1}}\left(0, \mu_{2}\right)=0$

$$
\begin{aligned}
& \left(\hat{\pi}_{-}\right)_{n+1, \ldots, 2 n}\left(0, \mathrm{y}^{\prime}(\mu) ; 0 ; \Lambda ; 0, \mu\right) \\
& =\left(\hat{\pi}_{-}\right)_{n+1, \ldots, 2 n}\left(0, \mathrm{y}^{\prime}\left(0, \mu_{2}\right) ; 0 ; 0 ; 0,0, \mu_{2}\right) \\
& \quad+\frac{\partial\left(\hat{\pi}_{-}\right)_{n+1, \ldots, 2 n}}{\partial \Lambda} \Lambda+\frac{\partial^{2}\left(\hat{\pi}_{-}\right)_{n+1, \ldots, 2 n}}{\partial \Lambda^{2}} \Lambda^{2} \\
& \quad+\frac{\partial\left(\hat{\pi}_{-}\right)_{n+1, \ldots, 2 n}}{\partial\left(x^{\prime}, y^{\prime}\right)}\left(0, \mathrm{y}^{\prime}\left(\mu_{1}, \mu_{2}\right)-\mathrm{y}^{\prime}\left(0, \mu_{2}\right)\right)+\frac{\partial\left(\hat{\pi}_{-}\right)_{n+1, \ldots, 2 n}}{\partial \mu_{1}} \mu_{1} \\
& \quad+O\left(\mu_{1}^{2}, \Lambda^{3}\right) \\
& =-\frac{1}{\lambda\left(0 ; 0,0, \mu_{1}\right)} \mu_{2} \Lambda+\frac{\partial^{2}\left(\hat{\pi}_{-}\right)_{n+1, \ldots, 2 n}}{\partial \Lambda^{2}} \Lambda^{2}-\mu_{1}+O\left(\mu_{1}^{2}, \Lambda^{3}\right)
\end{aligned}
$$

Also Hypothesis 7 implies that all other $\left(\hat{\pi}_{-}\right)_{i_{1}, \ldots, i_{n}}$ are also bounded for small $\mu_{1}$ and $\Lambda$.

Second, by lemma 4.5

$$
\begin{gathered}
\left(\hat{\pi}_{0}\right)_{1, n+2, \ldots, 2 n}\left(\xi\left(\bar{y}(\mu) ; 0, \mu_{1}, \mu_{2}\right), \eta_{1}\left(\bar{y}(\mu) ; 0, \mu_{1}, \mu_{2}\right), \bar{y}(\mu),\left(\hat{\pi}_{-}\right)_{n+1, \ldots, 2 n}, \ldots\right) \\
=\left(\left(\hat{\pi}_{-}\right)_{n+1, \ldots, 2 n}^{-1} \eta_{1}^{2 \frac{\lambda(\Lambda ; 0, \mu)}{\lambda(0 ; 0, \mu)}}, \ldots, Z_{i_{1}, \ldots, i_{n}}, \ldots\right)
\end{gathered}
$$

where

$$
\begin{aligned}
\left|Z_{i_{1}, \ldots, i_{n}}\right| & <\left(\hat{\pi}_{-}\right)_{n+1, \ldots, 2 n}^{-1} \cdot\left(\hat{\pi}_{-}\right)_{i_{1}, \ldots, i_{n}} \cdot \eta_{1}^{\frac{\lambda(\Lambda ; 0, \mu)+\nu}{\lambda(0 ; 0, \mu)}} \\
& =\left(\hat{\pi}_{0}\right)_{1, n+2, \ldots, 2 n} \cdot\left(\hat{\pi}_{-}\right)_{i_{1}, \ldots, i_{n}} \cdot \eta_{1}^{\frac{\nu}{\lambda}-1}
\end{aligned}
$$

for $\left(i_{1}, \ldots, i_{n}\right) \neq(n+1, \ldots, 2 n)$.

Third, by lemmas $4.6,4.7,4.8$ and 4.9 , and the facts that $\mathrm{y}^{\prime}\left(0, \mu_{1}\right)=\xi\left(0 ; 0,0, \mu_{1}\right)$, $\frac{\partial \bar{y}}{\partial \mu_{1}}\left(0, \mu_{2}\right)=0$ and $\eta_{1}\left(0 ; 0, \mu_{1}, \mu_{2}\right)=\mu_{1}+O\left(\mu_{1}^{2}\right)$ 


$$
\begin{aligned}
& \left(\hat{\pi}_{+}\right)_{n+1, \ldots, 2 n}\left(\eta_{1}\left(\bar{y}(\mu) ; 0, \mu_{1}, \mu_{2}\right), \bar{y}(\mu), \xi\left(\bar{y}(\mu) ; 0, \mu_{1}, \mu_{2}\right) ; Z ; \Lambda ; 0, \mu_{1}, \mu_{2}\right) \\
& =\left(\hat{\pi}_{-}\right)_{n+1, \ldots, 2 n}\left(\eta_{1}\left(\bar{y}(\mu) ; 0, \mu_{1}, \mu_{2}\right), \bar{y}(\mu), \xi\left(\bar{y}(\mu) ; 0, \mu_{1}, \mu_{2}\right) ; Z ; \Lambda ; 0,-\mu_{1}, \mu_{2}\right) \\
& =\left(\hat{\pi}_{-}\right)_{n+1, \ldots, 2 n}\left(0,0, \xi\left(0 ; 0,0, \mu_{2}\right) ; 0 ; 0 ; 0,0, \mu_{2}\right) \\
& \quad+\frac{\partial\left(\hat{\pi}_{-}\right)_{n+1, \ldots, 2 n}}{\partial \Lambda} \Lambda+\frac{\partial^{2}\left(\hat{\pi}_{-}\right)_{n+1, \ldots, 2 n}}{\partial \Lambda^{2}} \Lambda^{2} \\
& \quad+\frac{\partial\left(\hat{\pi}_{-}\right)_{n+1, \ldots, 2 n}}{\partial\left(x^{\prime}, y^{\prime}\right)}\left(\eta_{1}\left(\bar{y}(\mu) ; 0, \mu_{1}, \mu_{2}\right), \bar{y}(\mu), \xi\left(\bar{y}(\mu) ; 0, \mu_{1}, \mu_{2}\right)-\xi\left(0 ; 0,0, \mu_{2}\right)\right) \\
& \quad-\frac{\partial\left(\hat{\pi}_{-}\right)_{n+1, \ldots, 2 n}}{\partial \mu_{1}} \mu_{1}+\frac{\partial\left(\hat{\pi}_{-}\right)_{n+1, \ldots, 2 n}}{\partial Z} Z+O\left(\mu_{1}^{2}, \Lambda^{3}, Z^{2}\right) \\
& \quad-\frac{1}{\lambda\left(0 ; 0,0, \mu_{1}\right)} \mu_{2} \Lambda+\frac{\partial^{2}\left(\hat{\pi}_{-}\right)_{n+1, \ldots, 2 n} \Lambda^{2}}{\partial \Lambda^{2}} \\
& \quad+\frac{\partial\left(\pi_{-}\right)_{n+1, \ldots, 2 n}}{\partial x_{1}^{\prime}} \mu_{1} \\
& \quad+\mu_{1}-Z_{1, n+2, \ldots, 2 n} \\
& \quad+O\left(Z_{i_{1}, \ldots, i_{n}}, \mu_{1}^{2}, \Lambda^{3},\right)
\end{aligned}
$$

Therefore

$$
\begin{aligned}
& \left(\hat{\pi}_{+} \circ \hat{\pi}_{0} \circ \hat{\pi}_{-}\right)_{n+1, \ldots, 2 n}\left(0, \mathrm{y}^{\prime}(\mu) ; 0 ; \Lambda ; 0, \mu\right) \\
& =-\frac{1}{\lambda\left(0 ; 0,0, \mu_{1}\right)} \mu_{2} \Lambda+\frac{\partial^{2}\left(\hat{\pi}_{-}\right)_{n+1, \ldots, 2 n}}{\partial \Lambda^{2}} \Lambda^{2}+\frac{\partial\left(\pi_{-}\right)_{n+1, \ldots, 2 n}}{\partial x_{1}^{\prime}} \mu_{1}+\mu_{1} \\
& -\frac{\mu_{1}^{2 \frac{\lambda(\Lambda ; 0, \mu)}{\lambda(0 ; 0, \mu)}}}{-\frac{1}{\lambda\left(0 ; 0,0, \mu_{1}\right)} \mu_{2} \Lambda+\frac{\partial^{2}\left(\hat{\pi}_{-}\right)_{n+1, \ldots, 2 n}}{\partial \Lambda^{2}} \Lambda^{2}-\mu_{1}}+O\left(\mu_{1}^{\min \left\{2, \frac{\nu}{\lambda}\right\}}, \Lambda^{3}\right)
\end{aligned}
$$

Let $E(\Lambda, \mu)$ be defined by the right hand side of (4.13). Then, by lemma $4.3, \Lambda$ is an eigenvalue if $E(\Lambda, \mu)=0$. Here, as $\Lambda=0$ is an eigenvalue and the eigenfunction is given by the $s$ derivative of the wave, $E(0, \mu)=0$ and thus

$$
\frac{\partial\left(\pi_{-}\right)_{n+1, \ldots, 2 n}}{\partial x_{1}^{\prime}} \mu_{1}=-2
$$

The existence of pure imaginary eigenvalues is proven by a scaling argument. Let $\mu_{2}=\sqrt{\mu_{1}} \tilde{\mu}_{2}$ and $\Lambda=\sqrt{\mu_{1}} \tilde{\Lambda}$, and consider the following:

$$
\begin{aligned}
\tilde{E}\left(\tilde{\Lambda}, \mu_{1}, \tilde{\mu}_{2}\right):= & \frac{1}{\mu_{1}} E(\Lambda, \mu) \\
= & -\frac{1}{\lambda\left(0 ; 0,0, \mu_{1}\right)} \tilde{\mu}_{2} \tilde{\Lambda}+\frac{\partial^{2}\left(\hat{\pi}_{-}\right)_{n+1, \ldots, 2 n}}{\partial \Lambda^{2}} \tilde{\Lambda}^{2}-1 \\
& \quad-\frac{\mu_{1}^{2 \frac{\lambda(\Lambda, 0, \mu)}{\lambda(0 ; 0, \mu)}-2}}{-\frac{1}{\lambda\left(0 ; 0,0, \mu_{1}\right)} \tilde{\mu}_{2} \tilde{\Lambda}+\frac{\partial^{2}\left(\hat{\pi}_{-}\right)_{n+1, \ldots, 2 n}}{\partial \Lambda^{2}} \tilde{\Lambda}^{2}-1}+O\left(\mu_{1}^{\min \left\{1, \frac{\nu}{\lambda}-1\right\}}, \sqrt{\mu_{1}} \tilde{\Lambda}^{3}\right)
\end{aligned}
$$


$\tilde{E}(\Lambda, \mu)$ can be continuously extended to $\mu_{1}=0$. The equation:

$$
\begin{aligned}
\lim _{\mu_{1} \downarrow 0} \tilde{E}\left(\tilde{\Lambda}, \mu_{1}, \tilde{\mu}_{2}\right) & =-\frac{1}{\lambda\left(0 ; 0,0, \mu_{1}\right)} \tilde{\mu}_{2} \tilde{\Lambda}+\hat{\pi}_{\Lambda \Lambda} \tilde{\Lambda}^{2}-1-\frac{1}{-\frac{1}{\lambda\left(0 ; 0,0, \mu_{1}\right)} \tilde{\mu}_{2} \tilde{\Lambda}+\hat{\pi}_{\Lambda \Lambda} \tilde{\Lambda}^{2}-1} \\
& =0
\end{aligned}
$$

has solutions

$$
\tilde{\Lambda}=0, \frac{\tilde{\mu}_{2}}{\lambda \hat{\pi}_{\Lambda \Lambda}} \quad \text { and } \quad \frac{\tilde{\mu}_{2} \pm \sqrt{\tilde{\mu}_{2}^{2}+8 \hat{\pi}_{\Lambda \Lambda} \lambda^{2}}}{2 \lambda \pi_{\Lambda \Lambda}}
$$

where $\hat{\pi}_{\Lambda \Lambda}$ stands for $\frac{\partial^{2}\left(\hat{\pi}_{-}\right)_{n+1, \ldots, 2 n}}{\partial \Lambda^{2}}$. The last two solutions are pure imaginary when $\tilde{\mu}_{2}=0$; therefore, the equation $\tilde{E}\left(\tilde{\Lambda}, \mu_{1}, \tilde{\mu}_{2}\right)=0$ has pure imaginary solutions for small $\mu_{1}>0$, which are near $\pm \sqrt{\frac{2}{\hat{\pi}_{\Lambda \Lambda}}}$, because $\tilde{E}$ is continuous for $\mu_{1} \geq 0$ and analytic in $\Lambda$ near $\frac{\tilde{\mu}_{2} \pm \sqrt{\tilde{\mu}_{2}^{2}+8 \hat{\pi}_{\Lambda \Lambda} \lambda^{2}}}{2 \lambda \pi_{\Lambda \Lambda}}$. This proves the theorem.

5. Discussion. First, notice that the bifurcation curve $\mu_{1}=\phi\left(0, \mu_{2}\right)$ for $c=0$ in theorem 2 is that of a pitchfork bifurcation of the standing pulse. This is easily seen from the following two facts. The first is that

$$
\begin{aligned}
& \tilde{H}\left(c, \mu_{1}, \mu_{2}\right) \\
& \quad=\eta_{1}\left(Y\left(c, \mu_{1}, \mu_{2}\right), c, \mu_{1}, \mu_{2}\right)^{\lambda\left(c, \mu_{1}, \mu_{2}\right)}-\eta_{1}\left(X\left(c, \mu_{1}, \mu_{2}\right),-c, \mu_{1}, \mu_{2}\right)^{\lambda\left(-c, \mu_{1}, \mu_{2}\right)}
\end{aligned}
$$

measures the separation of the stable and unstable manifolds $W^{s}(Q)$ and $W^{u}(Q)$ of $Q$. The second is that $\tilde{H}\left(0, \mu_{1}, \mu_{2}\right)=0$ and $\frac{\partial}{\partial c} \tilde{H}\left(0, \mu_{1} \mu_{2}\right)=H\left(0, \mu_{1} \mu_{2}\right)=0$ mean that that Evans' function $E(\Lambda)$ for the pulse has a double zero at the origin.

Next, as is mentioned in the introduction, the theorems of this paper apply to the following $\varepsilon-\tau$ system treated in [13] and [8]:

$$
\left\{\begin{aligned}
\varepsilon \tau u_{t} & =\varepsilon^{2} u_{x x}+f(u, v ; \theta, \gamma) \\
v_{t} & =v_{x x}+g(u, v ; \theta, \gamma)
\end{aligned}\right.
$$

[13] proves that the hypotheses in this paper are satisfied for suitably chosen $f$ and $g$, but it only proves the existence of pulses bifurcating from transversal intersection of $M_{1}$ and $M_{2}$.

[8] proves the existence of pitchfork and Hopf bifurcations for piece-wise linear $f$ and $g$ by the singular perturbation technique. Although their results exclude a small neighborhood around the degenerate point in the parameter space because of lack of transversality, the asymptotic of the bifurcation curve of pitchfork bifurcation for the singular limit system agree with that of theorem 1 of this paper.

The order of the bifurcations, that is, whether Hopf bifurcation or pitchfork bifurcation occurs first when $\mu_{2}$ is decreased, is not determined by the analysis of this paper.

\section{REFERENCES}

[1] J. Alexander, R. Gardner, and C. Jones, A topological invariant arising in the stability analysis of travelling waves, J. Reine Angew. Math., 410 (1990), pp. 167-212. 
[2] J. AleXANDER AND C. Jones, Existence and stability of asymptotically oscillatory double pulses, J. Reine Angew. Math., 446 (1994), pp. 49-79.

[3] —- Existence and stability of asymptotically oscillatory triple pulses, Z. Angew. Math. Phys., 44 (1993), pp. 189-200.

[4] B. Deng, The existence of infinitely many traveling front and back waves in the FitzHughNagumo equations, SIAM J. Math. Anal., 22 (1991), pp. 1631-1650.

[5] J. Evans, N. FEnIChel, AND A. FERoe, Double impulse solutions in nerve axon equations, SIAM J. Appl. Math., 42 (1982), pp. 219-234.

[6] R. GARDNER, Instability of oscillatory shock profile solutions of the generalized Burgers-KdV equation, Physica D, 90 (1996), pp. 366-386.

[7] D. HENRY, The geometric theory of semilinear parabolic equations, Lec. Notes in Math. 840, Springer, 1981.

[8] T. IKEDA, H. IKEDA, AND M. Mimura, Hopf bifurcation of travelling pulses in some bistable reaction-diffusion systems, Methods Appl. Anal., 7 (2000), pp. 165-193.

[9] T. Kato, Perturbation Theory for Linear Operators, Springer, 1976.

[10] Y. KAN-ON, Existence of standing waves for competition-diffusion equations, Japan J. Indust. Appl. Math., 13 (1996), pp. 117-133.

[11] Y. KAN-ON, Instability of stationary solutions for Lotka-Volterra competition model with diffusion, J. Math. Anal. Appl., 208 (1997), pp. 158-170.

[12] H. KoKUBU, Homoclinic and heteroclinic bifurcation of vector fields, Japan J. Appl. Math., 5 (1988), pp. 455-501.

[13] H. KoKubu, Y. Nishiura, AND H. OKa, Heteroclinic and homoclinic bifurcations in bistable reaction diffusion systems, J. Diff. Eqs., 86 (1990), pp. 260-341.

[14] S. NII, An extension of the stability index for travelling wave solutions and its application for bifurcations, SIAM J. Math. Anal., 28 (1997), pp. 402-433.

[15] — Stability of travelling multiple-front (multiple-back) wave solutions of the FitzHughNagumo Equations, SIAM J. Math. Anal., 28 (1997), pp. 1094-1112.

[16] A topological proof of stability of $N$-front solutions of the FitzHugh-Nagumo Equations, J. Dyn. Diff. Eqns, 11 (1999), pp. 515-555.

[17] S .NiI AND B. Sandstede, in preparation.

[18] B. SAndstede, Stability of multiple-pulse solutions, Trans. Amer. Math. Soc., 350 (1998), pp. 429-472.

[19] - Stability of $N$-fronts bifurcating from a twisted heteroclinic loop and an application to the FitzHugh-Nagumo equation, SIAM J. Math. Anal., 29 (1998), pp. 183-207.

[20] B. Sandstede, J. Alexander, And C. Jones, Existence and stability of n-pulses on optical fibers with phase-sensitive amplifiers, Physica D, 106 (1997), pp. 167-206.

[21] E. YANAGIDA, Branching of double-pulse solutions from single pulse solutions in nerve axon equations, J. Diff. Eqs., 66 (1987), pp. 243-262.

[22] E. YANAGIDA AND K. MAGINU, Stability of double-pulse solutions in nerve axon equations, SIAM J. Appl. Math., 49 (1989), pp. 1158-1173.

[23] A. YEw, Analytical Study of Solitary-Waves in Quadratic Media, PhD thesis, Brown University, 1998. 
S. NII 